\title{
Trasmisión de Volatilidad del COVID-19 a los Precios de Acciones del Sector Bancario e Industrial de Sudamérica, México y Estados Unidos
}

\section{Transmission of Volatility of COVID-19 to the Share Prices of the Banking and Industrial Sectors of South America, Mexico and the United States}

\author{
Luis Eduardo Peñafiel ${ }^{1}$ https:///orcid.org/0000-0002-5571-3978 \\ ${ }^{1}$ Pontificia Universidad Católica Argentina, Buenos Aires, Argentina \\ lueduardo1994@gmail.com
}

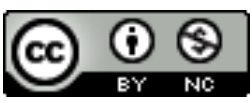

Esta obra está bajo una licencia internacional

Creative Commons Atribución-NoComercial 4.0. $\begin{array}{ll}\text { Enviado: } & 2021 / 01 / 20 \\ \text { Aceptado: } & 2021 / 04 / 07 \\ \text { Publicado: } & 2021 / 06 / 30\end{array}$

\section{Resumen}

Este trabajo tiene como objetivo la modelización de la volatilidad de los precios de acciones del sector bancario e industrial de Sudamérica, México y Estados Unidos en el contexto de la pandemia del COVID-19. Para ello, se estiman los modelos TGARCH $(1,1)$ y EGARCH $(1,1)$. Estos modelos empíricos son ventajosos, porque permiten una respuesta asimétrica de la varianza condicionada en función del signo de los residuos. Es un elemento crucial para los diseños de cartera y gestión de riesgos, y la medición de la volatilidad de los mercados financieros para capturar potenciales oportunidades o pérdidas de inversión. El análisis realizado revela que Brasil fue el más volátil ante el impacto del COVID-19; lo siguen Colombia, Perú, Chile y Argentina. Un caso aparte son Ecuador y México, que no tuvieron impacto significativo en la volatilidad de los precios de las acciones. Por otro lado, el shock del COVID-19 ha impactado en mayor medida a la volatilidad del sector industrial en Sudamérica y México comparado con el sector bancario en los Estados Unidos.

Palabras clave: heterocedasticidad condicional, volatilidad, precios, COVID-19.

\begin{abstract}
This work is aimed at modeling the volatility of share prices in the banking and industrial sectors of South America, Mexico, and the United States during the COVID-19 pandemic. For this purpose, the TGARCH $(1,1)$ and EGARCH $(1,1)$ models are estimated. These empirical models are advantageous because they allow an asymmetric response of the conditioned variance depending on the
\end{abstract}

Sumario: 1 Introducción, 2 Marco Teórico, 3 Metodología, 4 Resultados, 5 Conclusiones.

Como citar: Peñafiel, L. E. (2021). Trasmisión de volatilidad del covid-19 a los precios de acciones del sector bancario e industrial de Sudamérica, México y Estados Unidos. Revista Tecnológica - Espol, 33(1), 105-125. http://www.rte.espol.edu.ec/index.php/tecnologica/article/view/802 
sign of the residuals. It is a crucial element for portfolio designs and risk management to measure the volatility of financial markets and capture potential investment opportunities or losses. The analysis carried out reveals that Brazil was the most volatile when facing the impact of COVID-19, followed by Colombia, Peru, Chile, and Argentina. A separate case is Ecuador and Mexico, which did not have a significant impact on the volatility of share prices. On the other hand, the COVID-19 shock had a greater impact on the volatility of the industrial sector in South America and Mexico, while affecting greatly the banking sector in the United States.

Keywords: Conditional heteroskedasticity, volatility, prices, COVID-19.

\section{Introducción}

Cada vez más los pronósticos indican que los efectos de la pandemia COVID-19 superarán a las crisis financieras pasadas (Peñafiel Chang, et al. 2020; Peñafiel Chang, 2021). En principio, el radical confinamiento se ha manifestado en una clara externalidad negativa que ha provocado tres tipos de shock simultáneos. Por un lado, está el shock de demanda causado por el hecho de que las empresas se ven forzadas a realizar reestructuraciones (Peñafiel Chang, 2020a); esto debido a que, a diferencia de otras crisis, en esta no podrán seguir produciendo como lo hacían antes, a excepción de algunas industrias específicas.

Por otro lado, está el shock de oferta que se produce por el periodo de deflación originado por la reducción del gasto de las familias (Peñafiel Chang, 2020b), y, finalmente, el shock financiero, producido por la suma de los dos shocks anteriores, que se caracteriza por el aumento de la morosidad debido a las dificultades en la devolución de las deudas (Peñafiel Chang, 2019; Peñafiel Chang \& Camelli, 2020). Este estudio se centra en los efectos sobre los mercados financieros, aunque estos diversos shocks afectan a muchos sectores de la economía de manera distinta.

Y es que, con la llegada de la pandemia, los mercados financieros de todo el mundo han tenido fluctuaciones en los precios de sus activos; fenómeno que se describe como un incremento de la volatilidad (Chaudhary, Bakhshi, \& Gupta, 2020; Chevallier, 2020). De forma que, tanto para los participantes del mercado financiero como para los reguladores, es fundamental poseer y entender los indicadores de volatilidad para identificar potenciales oportunidades o pérdidas de inversión. Para ello, esta investigación revisa un conjunto de medidas de volatilidad aplicadas a los precios de acciones del sector industrial y bancario de Sudamérica, México y Estados Unidos.

Qué mercados financieros han sido más volátiles en el contexto del COVID-19 es la pregunta a la que se trata de responder en esta investigación. Más, precisamente, este trabajo indaga de modo empírico sobre el rol que ha tenido la pandemia en la volatilidad de los mercados financieros de Sudamérica, México y Estados Unidos. Para ello, se estiman los modelos TGARCH $(1,1)$ y EGARCH $(1,1)$; estos modelos empíricos son ventajosos, porque permiten una respuesta asimétrica de la varianza condicionada en función del signo de los residuos.

A parte de esta primera sección que corresponde a la introducción y que ha explorado a groso modo el estado actual de la problemática planteada, el trabajo está ordenado del siguiente modo: la sección dos revisa la literatura respecto con la volatilidad de los mercados financieros; luego, en la sección tres, se presenta un análisis econométrico formal, cuyos resultados se exponen de modo resumido en la sección cuatro; para finalizar, se presentan las conclusiones en la quinta sección.

\section{Marco Teórico}

La definición de volatilidad en los mercados financieros se asocia a la amplitud de las fluctuaciones de los rendimientos de un activo en el tiempo, y aunque se trata de un concepto muy intuitivo, no está exento de dificultades (Fernández, 2002). La modelización de la volatilidad asociada a un activo financiero ha crecido exponencialmente y ha dado lugar a un gran volumen de investigaciones (Bollerslev, Chou, y Kroner, 1992; Christoffersen y Diebold, 1997; Begoña, 1998; Amigo Dobaño, 1999; Claessen y Mittnik, 2002; Poon y Granger, 2003; Engle, 2004; Figlewski, 2004; 
Frömmel, 2004; Poon, 2005; Knight y Satchell, 2007; Montenegro, 2010; Opschoor, 2013; DattaChaudhuri y Ghosh, 2015; Rupande, Muguto y Muzindutsi, 2019; Pflueger, Siriwardane y Sunderam, 2019; Bhowmik y Wang, 2020; Rcolani y Natoli, 2020).

La literatura considera a la volatilidad como un proceso que evoluciona de manera aleatoria en el tiempo. Por ello, la mayoría de los modelos planteados para captar el comportamiento de la volatilidad se formulan sobre alguna medida de dispersión, cuya desviación típica $\left(\sigma_{t}\right)$ es alguna transformación, siendo en esta lo más frecuente $\sigma_{t}^{2} \mathrm{y} \ln \sigma_{t}^{2}$.

Las diferentes maneras de representar esta variable surgen como respuesta a la relación existente entre la volatilidad y las variables del conjunto de información. Generalmente, son las novedades sobre su valor fundamental y las alteraciones en el entorno macroeconómico las principales fuentes de cambios en los precios de los activos (Kim y Wu, 1987; Cooper, Chuin y Atkin, 2004; Bilson, Brailsford, y Hooper, 2005; Cauchie, Hoesli, y Isakov, 2005; Flannery y Protopapadakis, 2005; Baresa, Bogdan, y Ivanovic, 2013; Ato Forson y Janrattanagul, 2014; Barnor, 2014; Fama, 2014; Jakpar, Tinggi, Tak, y Chong, 2018; Demir, 2019; Rehman, Kashif, y Saleem, 2019).

Sin embargo, al existir comúnmente problemas de concordancia entre la frecuencia de las variables financieras respecto con las variables macroeconómicas, la opción más utilizada por la literatura es la información de la propia historia de la serie. La evidencia del conjunto de trabajos revisados también muestra que la capacidad de modelización de la volatilidad depende del mercado analizado y de las medidas del error de previsión. Por consiguiente, la mejor manera de medir la volatilidad vendrá dado por cómo se tenga en cuenta aspectos del mercado en concreto, la periodicidad de las observaciones y el uso final de la previsión de la volatilidad.

En ese sentido, los modelos tipo GARCH han sido fuertemente impulsados al adaptarse con facilidad a las dinámicas antes mencionadas, porque permiten diferentes parametrizaciones de la ecuación de la varianza. Sobre esto existen estudios relevantes (Alonso, 1992; Goyal, 2000; Gazda y Výrost, 2003; Alberg, Shalit, y Yosef, 2008; Wu, 2010; Panait, 2012; Ali, 2013; Caldeira, Machado, Souza, y Tanscheit, 2014; Lama, Jha, Paul, y Gurung, 2015; Petrica y Stancu, 2017; Sosa, Bucio, y Cabello, 2019; Hanapi et al., 2020; Li, Clements, y Drovandi, 2020).

De hecho, diversos trabajos, como los de Bhunia y Ganguly (2020); Bildirici, Bayazit, y Ucan (2020); Duttilo, Gattone y Battista (2021); Fakhfekh, Jeribi y Ben Salem (2021); Lamouchi y Badkook, 2020; Ngu Chuan Yong, Mahdi Ziaei, y R. Szulczyk (2021), han incluido dentro de sus análisis los modelos tipo GARCH para conocer la volatilidad de los mercados financieros durante el estallido del nuevo coronavirus. Por tal motivo, el interés de este trabajo se centra en los modelos GARCH asimétricos TGARCH y EGARCH, debido a que se requiere la introducción de la posibilidad de que la volatilidad responda de manera asimétrica ante cambios de distintos signos en los precios.

\section{Metodología}

En esta sección se reportan las estimaciones formales de los parámetros de las especificaciones de $(\varphi, \theta, \eta, \lambda)$ de los modelos EGARCH y TGARCH. El razonamiento de colección de las variables obedeció al interés de examinar el impacto de la pandemia a la volatilidad de precios de las acciones del sector industrial y bancario. La muestra que se analizó estuvo compuesta por observaciones diarias entre el 5 de enero hasta el 20 de mayo de 2020, obtenidas de las bolsas de valores de cada país.

El estudio consideró el análisis de las acciones con mayor presencia bursátil, es decir, las acciones con mayor grado de liquidez. Se lo calculó dividiendo el número de negociaciones en que la acción ha sido transada en un tiempo determinado para el numero de negociaciones realizadas en total, durante el mismo periodo de tiempo. Para este estudio se consideró las acciones de los siguientes países: Estados Unidos: JPMorgan Chase, Walmart, General Motors; Ecuador: Banco de Guayaquil, Corporación la Favorita, Natluk; Perú: Banco BBVA, Empresa Siderúrgica del Perú, Unión Andina de Cementos; Colombia: Bancolombia, Grupo Argos, Grupo Nutresa; Argentina: Grupo Supervielle, Cablevisión, Aluar Aluminio; Chile: Banco Itaú, Falabella, Embotelladora Andina; México: Grupo 
Financiero Inbursa, Grupo Bimbo, América móvil; Brasil: Banco Bradesco, Metalúrgica Gerdau, Atacadao.

Con el fin de analizar el impacto de la pandemia en los mercados financieros, se consideraron los modelos EGARCH y TGARCH, porque son capaces de modelar los cambios asimétricos de los incrementos y decrementos de los rendimientos de las acciones. Dicho de otro modo, permite captar la volatilidad de manera asimétrica ante cambios de distintos signos en los precios.

Un modelo EGARCH (modelo exponencial generalizado autoregresivo condicionalmente heterocedástico), desarrollado inicialmente por Nelson (1991), tiene la virtud de modelar el comportamiento asimétrico de la varianza, que depende del tiempo, y, al mismo momento, garantizar que la varianza sea siempre positiva.

Esta técnica modela el efecto de la asimetría al considerar una función g de las innovaciones $z_{-} t$, que son variables independientes e idénticamente distribuidas (i.i.d.) de media cero, que involucra tanto el valor de la innovación $z_{-} t$ como su magnitud expresada por medio de $\left|z_{t}\right|-E\left(\left|z_{t}\right|\right)$. Siguiendo la metodología propuesta por Monsegny y Cuervo (2008), en términos matemáticos, tenemos:

$$
\mathrm{g}\left(z_{t}\right)=(\theta+\lambda) z_{t}-\lambda \mathrm{E}\left(\left|z_{t}\right|\right)
$$

Donde $\theta$ y $\lambda$ son números reales. El efecto de asimetría puede verse claramente al expresar la función g por casos:

$$
\mathrm{g}\left(z_{t}\right)=\left\{\begin{array}{lll}
(\theta+\lambda) z_{t}-\lambda E\left(\left|z_{t}\right|\right) & \text { si } \quad z_{t} \geq 0 \\
(\theta-\lambda) z_{t}-\lambda E\left(\left|z_{t}\right|\right) & \text { si } \quad z_{t} \leq 0
\end{array}\right.
$$

Con esta definición de g, decimos que un proceso estocástico $\left\{y_{t}\right\}_{t \varepsilon T}$ obedece a un modelo en regresión exponencial generalizado autoregresivo condicionalmente heterocedástico de órdenes p y q, $\operatorname{EGARCH}(p, q)$, si satisface:

$$
\begin{gathered}
y_{t} \mid \psi_{t-1} \sim N\left(u_{t}, h_{t}\right) \\
u_{t}=\mathrm{E}\left[y_{t} \mid \psi_{t-1}\right] \\
=x_{t} \beta \\
\operatorname{Ln}\left(h_{t}\right)=\operatorname{Ln} \operatorname{VAR}\left[y_{t} \mid \psi_{t-1}\right] \\
=C+\sum_{k=1}^{\infty} \psi_{k} g\left(\varepsilon_{t-k}\right) \\
\varepsilon_{t}=y_{t}-x_{t} B
\end{gathered}
$$

Donde:

$\mathrm{y}_{\mathrm{t}}$ : variable aleatoria

$\mathrm{T}$ : es un conjunto discreto de índices

$\psi_{\mathrm{t}-1}$ : información pasada

$\mathrm{x}_{\mathrm{t}}$ : vector de observaciones de las variables explicativas

B: vector de parámetros desconocidos

$€$ : es una constante

$\psi_{k}$ : parámetros para la varianza que satisfacen la condición $\sum_{k=1}^{\infty}\left|\psi_{k}\right|^{2}<\infty$.

En la ecuación (3), si la media es cero, no se tiene el supuesto de normalidad, y los errores se definen como $\varepsilon_{t}=z_{t} h_{t}^{\frac{1}{2}}$, donde $\left\{z_{t}\right\} \mathrm{t} \varepsilon \mathrm{T}$ son variables i.i.d de media cero, varianza uno y tienen distribución del error generalizado con parámetro (v). El modelo EGARCH y la verosimilitud para T observaciones está dada por: 


$$
f\left(z_{t} \mid \psi_{t-1}\right)=\frac{(v) \exp \left(-\frac{1}{2}\left|z_{t} / \lambda\right|^{v}\right)}{\lambda 2^{\left(1+\frac{1}{v}\right)} \Gamma\left(\frac{1}{v}\right)}
$$

Donde $\Gamma$ (.) es la función gama,

$$
\lambda=\left[\frac{\Gamma\left(\frac{1}{v}\right)}{2\left(\frac{2}{v}\right) \Gamma\left(\frac{3}{v}\right)}\right]^{\frac{1}{2}}
$$

(v) es un parámetro positivo, y si es igual a 2, se tiene la distribución normal. A partir del logaritmo de la verosimilitud para T observaciones, se obtienen las estimaciones de los parámetros para la media y la varianza. La ecuación (5) puede expresarse como:

$$
\log \left(h_{t}\right)=\varphi+\sum_{i=1}^{q} \eta_{i}\left|\frac{u_{t-i}}{\sqrt{h_{t-i}}}\right|+\sum_{i=1}^{q} \lambda_{i} \frac{u_{t-i}}{\sqrt{h_{t-i}}}+\sum_{k=1}^{p} \theta_{k} \log \left(h_{t-k}\right)
$$

$\log \left(h_{t}\right)$ es el logaritmo de la serie de la variancia $\left(h_{t}\right)$, que hace que el efecto sea exponencial en vez de cuadrático. Esto asegura que las estimaciones no sean negativas. Donde, $\varphi=$ es una constante, $\eta=$ efectos Arch, $\lambda=$ efectos asimétricos, $\theta=$ efectos Garch. Si $\lambda_{1}=\lambda_{2}=\ldots=0$, el modelo es simétrico. Pero si $\lambda_{i}<0$, implica que shocks negativos generan mayor volatilidad que shocks positivos.

Por otro lado, un modelo TGARCH (modelo por umbrales generalizado autoregresivo, condicionalmente heterocedástico), desarrollado inicialmente por Zakoian (1994), tiene la virtud de dividir la distribución de los shocks en intervalos disjuntos. Dicho esto, un proceso estocástico $\left\{y_{t}\right\}_{t \varepsilon t}$, obedece a un modelo en regresión por umbrales generalizado autoregresivo condicionalmente heterocedástico de órdenes p y q, TGARCH (p, q), si satisface:

$$
\begin{gathered}
y_{t} \mid \psi_{t-1} \sim N\left(u_{t}, h_{t}\right) \\
u_{t}=\mathrm{E}\left[y_{t} \mid \psi_{t-1}\right] \\
=x_{t} \beta \\
h_{t}=\operatorname{VAR}\left[y_{t} \mid \psi_{t-1}\right] \\
=z_{t} \mathrm{~W} \\
=\varphi+\sum_{k=1}^{p} \theta_{k} h_{t-k}+\sum_{i=1}^{q} \eta_{i} u_{t-i}^{2}+\sum_{i=1}^{q} \lambda_{i} u_{t-i}^{2} D_{t-i}
\end{gathered}
$$

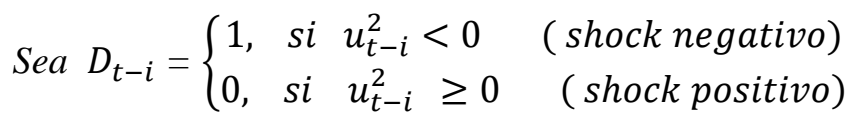

$$
\varepsilon_{t}=y_{t}-x_{t} \beta
$$

Donde:

$\mathrm{y}_{\mathrm{t}}$ : variable aleatoria

T: es un conjunto discreto de índices

$\psi_{\mathrm{t}-1}$ : información pasada

$\mathrm{x}_{\mathrm{t}}$ : vector de variables explicativas observadas en el tiempo $\mathrm{t}$.

$z_{t}$ : vector de variables para la varianza

$B$ : vector de parámetros para modelar la media 
W: vector de parámetros para modelar la varianza

$\varphi$ : constante

La variable $D_{i}$ usa un umbra igual a cero para clasificar los impactos pasados. En la ecuación (12), si el coeficiente $\lambda_{i}$, que corresponde al parámetro de apalancamiento, es cero, el modelo se convierte en un GARCH $(\mathrm{p}, \mathrm{q})$ estándar. De acuerdo con este modelo, cuando se tiene un shock positivo, el efecto que se tiene en la volatilidad es igual a $\eta_{i}$. Asimismo, cuando hay un shock negativo, el efecto en la volatilidad es $\left(\eta_{i}+\lambda_{i}\right)$. A partir del logaritmo de la verosimilitud para $\mathrm{T}$ observaciones, se obtienen las estimaciones de los parámetros para la media y la varianza. La ecuación (12) puede expresarse como:

$$
h_{t}=\varphi+\sum_{k=1}^{p} \theta_{k} h_{t-k}+\sum_{i=1}^{q}\left(\eta_{i}+\lambda_{i} D_{t-i}\right) u_{t-i}^{2}
$$

$\theta_{k}, \eta_{i}$ son parámetros no negativos que satisfacen condiciones similares a los modelos GARCH. Donde: $\varphi=$ es una constante, $\theta=$ efectos Garch, $\eta=$ efectos Arch, $\lambda=$ efectos asimétricos.

\section{Resultados}

Inminentemente entre el 5 de enero hasta el 20 de mayo de 2020 fue el periodo en que los gobiernos establecieron estrictos confinamientos en toda la región; acontecimiento que constituyó una clara externalidad negativa. En la sección de anexos, por una parte, se muestran las estimaciones de los modelos y la evolución de los precios (ver Figura 2) y por otra parte, en la misma sección, Figura 3, se presentan los rendimientos diarios de las acciones y, las pruebas de raíz unitarias (ver Tabla 9), que garantizan la estacionariedad de los datos. En cuanto a la estimación, solo se confirma que el shock de la pandemia afecta la variabilidad de los precios si y solo si ambos modelos (EGARCH $(1,1)$, TGARCH $(1,1)$ ) indican lo mismo; basta con que uno de ellos sea no significativo para dar por hecho que el efecto del COVID-19 no afectó a la volatilidad.

A continuación, en el histograma de la Figura 1 se muestra un resumen de las estimaciones de volatilidad de asimétrica $(\lambda)$ del modelo TGARCH $(1,1)$.

Figura 1

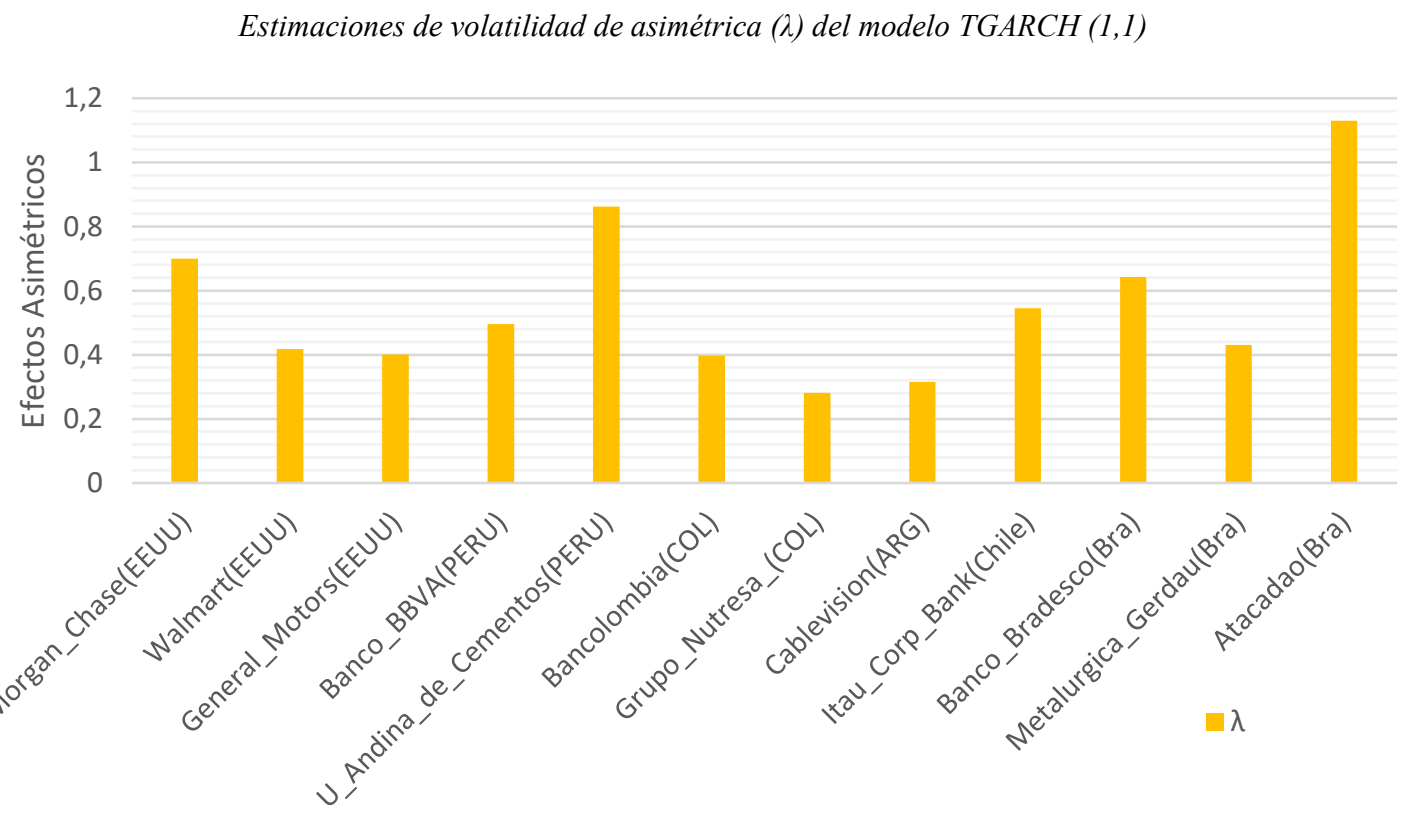

Notas. La figura muestra el coeficiente $(\lambda)$ que es la diferencia de volatilidad en la varianza condicional en condiciones normales con respecto al shock de la pandemia de los activos financieros. Con fines ilustrativos, se eligieron las acciones que dieron significativos en el modelo TGARCH $(1,1)$. Fuente: Bolsa de valores de cada país y elaboración propia. 
Por último, la elaboración y el diseño de este artículo responde a una minuciosa revisión de literatura que garantizó que la conjetura de investigación se cumpla. Siendo así, en este estudio se ha logrado construir modelos de la varianza condicional para las acciones con mayor presencia bursátil de cada sector de los siguientes países: Estados Unidos: JPMorgan Chase, Walmart, General Motors (ver Tabla 1); Ecuador: Banco de Guayaquil, Corporación la Favorita, Natluk (ver Tabla 2)Perú: Banco BBVA, Empresa Siderúrgica del Perú, Unión Andina de Cementos (ver Tabla 3); Colombia: Bancolombia, Grupo Argos, Grupo Nutresa (ver Tabla 4); Argentina: Grupo Supervielle, Cablevisión, Aluar Aluminio (ver Tabla 5); Chile: Banco Itaú, Falabella, Embotelladora Andina (ver Tabla 6); México: Grupo Financiero Inbursa, Grupo Bimbo, América móvil (ver Tabla 7); Brasil: Banco Bradesco, Metalúrgica Gerdau, Atacadao (ver Tabla 8).

\section{Conclusiones}

En este trabajo se muestra que la volatilidad de los activos financieros siempre ha sido un objeto de estudio de los inversionistas y organismos reguladores para poder realizar una valoración y gestión del riesgo más eficaz. Además, se analiza la influencia de la pandemia COVID-19 en la volatilidad de acciones del sector industrial y bancario de Sudamérica, México y Estados Unidos durante el periodo del 5 de enero de 2020 al 20 de mayo de 2020, mediante la estimación de los modelos EGARCH $(1,1)$ y TGARCH $(1,1)$.

Los hallazgos de este trabajo resultan interesantes en cuanto se evidencian cinco hechos estilizados para el mercado accionario de Sudamérica, México y Estados Unidos. Los resultados obtenidos muestran que: i) la pandemia no afectó significativamente a la volatilidad de las acciones de Ecuador y México; ii) la pandemia si afectó a la variabilidad de los precios de las acciones de: Chile (Banco Itau), Argentina (Cablevisión), Colombia (Bancolombia y Grupo Nutresa), Perú ( Unión Andina de Cementos y Banco BBVA), Brasil ( Banco Bradesco, Metalúrgica Gerdau y Atacadao), Estados Unidos ( JPMorgan Chase, Walmart, General Motors); iii) la volatilidad de Brasil es superior al resto de países; iv) a diferencia de Estados Unidos, en los demás países, en general, el shock de la pandemia afecta en mayor medida al sector industrial; v) el impacto de la pandemia en la volatilidad de los precios de las acciones de la región es bastante heterogénea, es decir, mientras que en Estados Unidos la pandemia afecta a la volatilidad de los precios de todas las empresas, para el caso de la región no aplica lo mismo.

Sería interesante en futuros estudios extender el análisis a un mayor número de acciones y otros tipos de activos financieros. Por otra parte, los resultados inevitablemente estarán sesgados por el criterio técnico del profesional, las decisiones impactarán en la elección de los métodos que capten el comportamiento de la volatilidad. En todo caso, la información obtenida de este estudio procura mostrar los efectos y las consecuencias de una pandemia a nivel global dentro del plan de acción de la política monetaria y administración de riesgos de los mercados de capitales.

\section{Referencias}

Alberg, D., Shalit, H., \& Yosef, R. (2008). Estimating stock market volatility using asymmetric GARCH models. Applied Financial Economics, 18(15), 1201-1208. https://doi.org/10.1080/09603100701604225

Ali, G. (2013). EGARCH, GJR-GARCH, TGARCH, AVGARCH, NGARCH, IGARCH and APARCH Models for Pathogens at Marine Recreational Sites. Journal of Statistical and Econometric Methods, 2(3), 1-6.

Alonso, F. (1992). La Volatilidad Del Mercado De Acciones Español. Retrieved from https://core.ac.uk/download/pdf/6501420.pdf

Baresa, S., Bogdan, S., \& Ivanovic, Z. (2013). Strategy of Stock Valuation By Fundamental Analysis. UTMS Journal of Economics, 4(1), 45-51.

Barnor, C. (2014). The impact of macroeconomic variables on stock market returns in Ghana (2000-2013). International Journal of Business and Commerce, 3(11), 1-31. 
Begoña, F. (1998). Modelización de series temporales financieras. Una recopilación. Statistics and Econometrics, 6(July), 1-46.

Bhowmik, R., \& Wang, S. (2020). Stock market volatility and return analysis: A systematic literature review. Entropy, 22(5), 1-18. https://doi.org/10.3390/E22050522

Bhunia, A., \& Ganguly, S. (2020). An assessment of volatility and leverage effect before and during the period of Covid-19: a study of selected international stock markets. International Journal of Financial Services Management, 10(2), 113. https://doi.org/10.1504/ijfsm.2020.110224

Bildirici, M., Bayazit, N. G., \& Ucan, Y. (2020). Analyzing crude oil prices under the impact of COVID-19 by using lstargarchlstm. Energies. https://doi.org/10.3390/en13112980

Bilson, C., Brailsford, T. J., \& Hooper, V. J. (2005). Selecting Macroeconomic Variables as Explanatory Factors of Emerging Stock Market Returns. SSRN Electronic Journal. https://doi.org/10.2139/ssrn.201908

Bollerslev, T., Chou, R. Y., \& Kroner, K. F. (1992). ARCH modeling in finance. A review of the theory and empirical evidence. Journal of Econometrics, 52(1-2), 5-59. https://doi.org/10.1016/03044076(92)90064-X

Caldeira, A. M., Machado, M. A. S., Souza, R. C., \& Tanscheit, R. (2014). Garch Model Indentification Using Neural Network. Independent Journal of Management \& Production, 5(2), 527-541. https://doi.org/10.14807/ijmp.v5i2.161

Cauchie, S., Hoesli, M. E. R., \& Isakov, D. (2005). The Determinants of Stock Returns in a Small Open Economy. SSRN Electronic Journal. https://doi.org/10.2139/ssrn.391996

Chaudhary, R., Bakhshi, P., \& Gupta, H. (2020). Volatility in International Stock Markets: An Empirical Study during COVID-19. Journal of Risk and Financial Management, 13(9), 208. https://doi.org/10.3390/jrfm13090208

Chevallier, J. (2020). COVID-19 Pandemic and Financial Contagion. Journal of Risk and Financial Management, 13(12), 309. https://doi.org/10.3390/jrfm13120309

Christoffersen, P. F., \& Diebold, F. X. (1997). How relevant is volatility forecasting for financial risk management? Review of Economics and Statistics, 82(1), 12-22. https://doi.org/10.1162/003465300558597

Claessen, H., \& Mittnik, S. (2002). Forecasting stock market volatility and the informational efficiency of the DAX-index options market. European Journal of Finance, 8(3), 302-321. https://doi.org/10.1080/13518470110074828

Cooper, R., Chuin, L., \& Atkin, M. (2004). Relationship between Macroeconomic Variables and Stock Market Indices: Cointegration Evidence from Stock Exchange of Singapore's All-S Sector Indices. Economics Letters, 21(3), 461-504. https://doi.org/10.1016/j.jfineco.2006.10.002

Datta, T., \& Ghosh, I. (2015). Forecasting Volatility in Indian Stock Market using Artificial Neural Network with Multiple Inputs and Outputs. International Journal of Computer Applications, 120(8), 7-15. https://doi.org/10.5120/21245-4034

Demir, C. (2019). Macroeconomic determinants of stock market fluctuations: The case of BIST-100. Economies, 7(1). https://doi.org/10.3390/economies7010008

Dobaño, L. (1999). Modelos Arch: Un análisis de volatilidad de series temporales financieras. Anuales de Estudios Económicos y Empresariales, (14), 67-84.

Duttilo, P., Gattone, S. A., \& Battista, T. Di. (2021). Volatility modeling : an overview of equity markets in the euro area during COVID-19 Pandemic. https://doi.org/10.3390/1010000

Engle, R. (2004). Risk and volatility: Econometric models and financial practice. American Economic Review, 
94(3), 405-420. https://doi.org/10.1257/0002828041464597

Fakhfekh, M., Jeribi, A., \& Ben Salem, M. (2021). Volatility dynamics of the Tunisian stock market before and during the COVID-19 outbreak: Evidence from the GARCH family models. International Journal of Finance and Economics, (February). https://doi.org/10.1002/ijfe.2499

Fama, E. F. (2014). Two pillars of asset pricing. American Economic Review, 104(6), 1467-1485. https://doi.org/10.1257/aer.104.6.1467

Fernández, M. D. R. (2002). Medidas de volatilidad. Revista Española de Financiación y Contabilidad, 31(114), 1073-1110. https://doi.org/10.1080/02102412.2002.10779469

Figlewski, S. (2004). Forecasting volatility. New York University Stern School of Business, 120-157.

Flannery, M. J., \& Protopapadakis, A. (2005). Macroeconomic Factors DO Influence Aggregate Stock Returns. SSRN Electronic Journal. https://doi.org/10.2139/ssrn.314261

Forson, J., \& Janrattanagul, J. (2014). Selected macroeconomic variables and stock market movements: Empirical evidence from Thailand. Contemporary Economics, 8(2), 157-174. https://doi.org/10.5709/ce.1897-9254.138

Frömmel, M. (2004). Modelling Exchange Rate Volatility in the Run-up to EMU using a Markov Switching GARCH Model. October, (306), 1-18.

Gazda, V., \& Výrost, T. (2003). Application of Garch Models in Forecasting the Volatility of the Slovak Share Index ( Sax ). Economics Focus, XI, 17-20.

Goyal, A. (2000). Predictability of Stock Return Volatility from GARCH Models, (May).

Hanapi, A. L. M., Othman, M., Sokkalingam, R., Ramli, N., Husin, A., \& Vasant, P. (2020). A novel fuzzy linear regression slidingwindow GARCH model for time-series forecasting. Applied Sciences (Switzerland), 10(6). https://doi.org/10.3390/app10061949

Jakpar, S., Tinggi, M., Tak, A. H., \& Chong, W. Y. (2018). Fundamental Analysis VS Technical analysis: The Comparison of Two Analysis in Malaysia Stock Market. UNIMAS Review of Accounting and Finance, 1(1), 38-61. https://doi.org/10.33736/uraf.1208.2018

Kim, M. K., \& Wu, C. (1987). Macro-Economic Factors and Stock Returns. Journal of Financial Research, 10(2), 87-98. https://doi.org/10.1111/j.1475-6803.1987.tb00481.x

Knight, J., \& Satchell, S. (2007). Forecasting Volatility In The Financial Markets. Quantitative Finance Series. https://doi.org/10.1016/B978-0-7506-6942-9.X5000-6

Lama, A., Jha, G. K., Paul, R. K., \& Gurung, B. (2015). Modelling and Forecasting of Price Volatility: An Application of GARCH and EGARCH Models. Agricultural Economics Research Review, 28(1), 73. https://doi.org/10.5958/0974-0279.2015.00005.1

Lamouchi, R. A., \& Badkook, R. (2020). Gold Prices Volatility among Major Events and During the Current COVID-19 Outbreak. Journal of Statistical and Econometric Methods, 9(July), 39-52.

Li, D., Clements, A., \& Drovandi, C. (2020). Efficient Bayesian estimation for GARCH-type models via Sequential Monte Carlo. Econometrics and Statistics, (Dan Li), 1-37. https://doi.org/10.1016/j.ecosta.2020.02.002

Nelson, D. (1991). Conditional Heteroskedasticity in Asset Returns. a New Approach. Econometrica 59(2): 347-370.

Ngu Chuan Yong, J., Mahdi Ziaei, S., \& R. Szulczyk, K. (2021). The Impact of Covid-19 Pandemic on Stock Market Return Volatility: Evidence from Malaysia and Singapore. Asian Economic and Financial Review, 11(3), 191-204. https://doi.org/10.18488/journal.aefr.2021.113.191.204 
Monsegny, M. C., \& Cuervo, E. C. (2008). Modelos arch, garch y egarch: Aplicaciones a series financieras. Cuadernos de Economia, 27(48), 287-319.

Montenegro, R. (2010). Medición de la volatilidad en series de tiempo financieras: Una evolución a la tasa de cambio representativa del mercado (TRM) en Colombia. Revista Finanzas y Política Económica, 2(1), $125-132$.

Opschoor, A. (2013). Understanding Financial Market Volatility. International Finance, 589-610. https://doi.org/10.1093/acprof:oso/9780199754656.003.0028

Panait, I. (2012). Using Garch-in-Mean Model to Investigate Volatility and Persistence at Different Frequencies for Bucharest Stock Exchange during 1997-2012. Theoretical and Applied Economics, 19(5), 55-76.

Peñafiel Chang, L. E. (2019). La Dinámica Macroeconómica y la morosidad del sistema financiero del Ecuador (2009-2018). Cuestiones Económica, 29(1). Retrieved from https://estudioseconomicos.bce.fin.ec/index.php/RevistaCE/article/view/35

Peñafiel Chang, L. E. (2020a). Decisiones empresariales de productividad y reajuste ante la crisis argentina 2018-2019. Caso GRIMOLDI. Ciencia UNEMI, 13(32), 109-122.

Peñafiel Chang, L. E. (2020b). Dinámica de la tasa de interés, deflación y producción ante el shock del coronavirus en el marco de un modelo 'DSGE' neo - keynesiano para la economía ecuatoriana. $X$ Pedientes Económicos, 4(10), 6-18. Retrieved from https://ojs.supercias.gob.ec/index.php/Xpedientes_Economicos/article/view/119

Peñafiel Chang, L. E. (2021). Regionalización de la cadena de valor: oportunidades y desafíos para América Latina en la nueva era del Sars-Cov-2 . Revista Ciencia UNEMI, 14, 81-91.

Peñafiel Chang, L. E., \& Camelli, G. B. (2020). Coordinación de Política Económica mediante el estudio de la Relación Dinámica-Macroeconómica de la cartera de morosidad de consumo del Ecuador en el periodo 2009-2019. Revista Tecnológica - ESPOL, 32(1). https://doi.org/10.37815/rte.v32n1.722

Peñafiel Chang, L. E., Camelli, G., \& Peñafiel Chang, P. (2020). Pandemia COVID-19: Situación política económica y consecuencias sanitarias en América Latina. Ciencia Unemi, 13(33), 120-128.

Petrica, A., \& Stancu, S. (2017). Empirical Results of Modeling EUR/RON Exchange Rate using ARCH, GARCH, EGARCH, TARCH and PARCH models. Romanian Statistical Review, 65(1), 57-72.

Pflueger, C., Siriwardane, E., \& Sunderam, A. (2019). Financial Market Risk Perceptions and the Macroeconomy*. The Quarterly Journal of Economics, 17-40. https://doi.org/10.1093/qje/qjaa009

Poon, S.-H. (2005). A Practical Guide to Forecasting Financial Market Volatility.

Poon, S.-H., \& Granger, W. (2003). Forecasting Volatility in financial markets: A Review. Journal of Economic Literature, 41, 478-539.

Rehman, N., Kashif, M., \& Saleem, A. (2019). Financial and Economic Indicators of economic advancement: Evidence from Asian countries. International Transaction Journal of Engineering, Management and Applied Sciences, 10(14), 1-10. https://doi.org/10.14456/ITJEMAST.2019.187

Rupande, L., Muguto, H. T., \& Muzindutsi, P. F. (2019). Investor sentiment and stock return volatility: Evidence from the Johannesburg Stock Exchange. Cogent Economics and Finance, 7(1), 1-16. https://doi.org/10.1080/23322039.2019.1600233

Sosa, M., Bucio, C., \& Cabello, A. (2019). Dependencia condicional en el bloque TLCAN : un análisis con modelos GARCH y Cópula. Ecos de Economía, 22(47), 73-91. https://doi.org/10.17230//ecos.2018.47.4

Wu, J. (2010). Threshold GARCH Model: Theory and Application. Publish.Uwo.Ca, (April), 1-42. Retrieved from http://publish.uwo.ca/ jwu87/files/Jing1207.pdf 
Zakoian, J. M., (1994.) Threshold Heteroskedastic Models. Journal of Economic Dynamics and Control.

\section{Anexos}

Tabla 1

Estimaciones de los parámetros y pruebas de diagnóstico para Estados Unidos

\begin{tabular}{|c|c|c|c|c|c|c|}
\hline & $\begin{array}{l}\text { JPMorgan Chase } \\
\text { EGARCH }(\mathbf{1 , 1})\end{array}$ & $\begin{array}{l}\text { JPMorgan Chase } \\
\text { TGARCH }(\mathbf{1 , 1})\end{array}$ & $\begin{array}{l}\text { Walmart } \\
\text { EGARCH }(1,1)\end{array}$ & $\begin{array}{l}\text { Walmart } \\
\text { TGARCH }(1,1)\end{array}$ & $\begin{array}{l}\text { General Motors } \\
\text { EGARCH }(\mathbf{1 , 1}) \\
\end{array}$ & $\begin{array}{l}\text { General Motors } \\
\text { TGARCH }(\mathbf{1 , 1})\end{array}$ \\
\hline \multirow[t]{2}{*}{$\mathrm{c}$} & $-0,0039$ & $-0,0052$ & $-0,0023$ & $-0,0019$ & $-0,0056$ & $-0,0034$ \\
\hline & {$[0,0782]$} & {$[0,0006]$} & {$[0,1037]$} & {$[0,1759]$} & {$[0,0514]$} & {$[0,1900]$} \\
\hline \multirow[t]{2}{*}{$\operatorname{Var}(-1)$} & $-0,1663$ & $-0,1709$ & $-0,1205$ & $-0,1574$ & 0,1520 & 0,1538 \\
\hline & {$[0,1002]$} & {$[0,0210]$} & {$[0,1998]$} & {$[0,0762]$} & {$[0,1577]$} & {$[0,1770]$} \\
\hline \multirow[t]{2}{*}{$\varphi$} & $-0,4322$ & 0,0000 & $-0,4982$ & 0,0000 & $-0,3533$ & 0,0000 \\
\hline & {$[0,0274]$} & {$[0,0009]$} & {$[0,0211]$} & {$[0,3755]$} & {$[0,0325]$} & {$[0,3802]$} \\
\hline \multirow[t]{2}{*}{$\eta$} & 0,1154 & $-0,2516$ & 0,3408 & 0,0474 & 0,1220 & $-0,0001$ \\
\hline & {$[0,6448]$} & {$[0,1040]$} & {$[0,0276]$} & {$[0,6468]$} & {$[0,5946]$} & {$[0,9993]$} \\
\hline \multirow[t]{2}{*}{$\lambda$} & $-0,3855$ & 0,6993 & $-0,2347$ & 0,4171 & $-0,3238$ & 0,4007 \\
\hline & {$[0,0032]$} & {$[0,0001]$} & {$[0,0270]$} & {$[0,0221]$} & {$[0,0177]$} & {$[0,0274]$} \\
\hline \multirow[t]{2}{*}{$\theta$} & 0,9520 & 0,9235 & 0,9652 & 0,8062 & 0,9559 & 0,8385 \\
\hline & {$[0,0000]$} & {$[0,0000]$} & {$[0,0000]$} & {$[0,0000]$} & {$[0,0000]$} & {$[0,0000]$} \\
\hline$R^{2}$ & 0,0960 & 0,0993 & 0,0473 & 0,0625 & $-0,0313$ & $-0,0293$ \\
\hline Akaike & $-4,1557$ & $-4,2272$ & $-5,0416$ & $-5,057$ & $-3,6943$ & $-3,6568$ \\
\hline Schwarz & $-3,9954$ & $-4,0669$ & $-4,8814$ & $-4,8969$ & $-3,5337$ & $-3,4963$ \\
\hline $\begin{array}{l}\text { Hannan- } \\
\text { Ouinn }\end{array}$ & $-4,0909$ & $-4,1623$ & $-4,0769$ & $-4,9924$ & $-3,6292$ & $-3,5920$ \\
\hline
\end{tabular}

Notas. Esta tabla muestra los resultados de regresión que provienen de la estimación de las ecuaciones (9) y (15), siendo el pvalue los valores que están en corchetes. La interpretación del parámetro de asimetría en los modelos EGARCH $(1,1)$ indican que si se satisface que $\lambda<0$ implica que un shock negativo genera mayor volatilidad que un shock positivo. Mientras que la interpretación del parámetro de asimetría en los modelos TGARCH $(1,1)$ indican que si se satisface que $\lambda>0$ implica que para ese activo hay asimetría en las noticias (Shock), es decir, que un shock negativo tiene mayores efectos de volatilidad que un shock positivo. Los datos de las acciones provienen de la bolsa de valores de Estados Unidos. Elaboración propia.

Para el caso de Estados Unidos, las estimaciones del parámetro $(\lambda)$ fueron significativas e indican que la pandemia afectó a la variabilidad de los precios de las acciones JPMorgan Chase, Walmart y General Motors. La estimación de la varianza condicional frente al shock de la pandemia y en condiciones normales queda construida como:

$$
\begin{aligned}
& \widehat{h_{t}} \text { JPMorgan }_{\text {Covid } 19}=0,000+0,9235 \widehat{h_{t-1}}+(-0,2561+0,6993) \widehat{u_{t-1}^{2}} \\
& \widehat{h_{t}} \text { JPMorgan }=0,000+0,9235 \widehat{h_{t-1}}+(-0,2561) \widehat{u_{t-1}^{2}} \\
& \widehat{h_{t}} \text { Walmart }_{\text {Covid } 19}=0,000+0,8062 \widehat{h_{t-1}}+(0,0474+0,4171) \widehat{u_{t-1}^{2}} \\
& \widehat{h_{t}} \text { Walmart }=0,000+0,8062 \widehat{h_{t-1}}+(0,0474) \widehat{u_{t-1}^{2}} \\
& \widehat{h_{t}} \text { General Motors }{ }_{\text {Covid } 19}=0,000+0,8385 \widehat{h_{t-1}}+(-0,0001+0,4007) \widehat{u_{t-1}^{2}} \\
& \widehat{h_{t}} \text { General Motors }=0,000+0,8385 \widehat{h_{t-1}}+(-0,0001) \widehat{u_{t-1}^{2}}
\end{aligned}
$$


Tabla 2

Estimaciones de los parámetros y pruebas de diagnóstico para Ecuador

\begin{tabular}{|c|c|c|c|c|c|c|}
\hline & $\begin{array}{l}\text { Banco de } \\
\text { Guayaquil } \\
\text { EGARCH }(1,1)\end{array}$ & $\begin{array}{l}\text { Banco de } \\
\text { Guayaquil } \\
\text { TGARCH }(1,1)\end{array}$ & $\begin{array}{l}\text { Corp. La } \\
\text { Favorita } \\
\text { EGARCH }(1,1)\end{array}$ & $\begin{array}{l}\text { Corp. La } \\
\text { Favorita } \\
\text { TGARCH }(1,1)\end{array}$ & $\begin{array}{l}\text { Natluk } \\
\text { EGARCH }(1,1)\end{array}$ & $\begin{array}{l}\text { Natluk } \\
\text { TGARCH (1,1) }\end{array}$ \\
\hline \multirow[t]{2}{*}{$\mathrm{C}$} & $-0,0032$ & $-0,0051$ & $-0,0001$ & $-0,0000$ & 0,0017 & 0,0002 \\
\hline & {$[0,0000]$} & {$[0,1196]$} & {$[0,7199]$} & {$[0,7923]$} & {$[0,0000]$} & {$[0,9056]$} \\
\hline \multirow[t]{2}{*}{$\operatorname{Var}(-1)$} & $-0,4490$ & $-0,4148$ & $-0,2501$ & $-0,2655$ & 0,2950 & $-0,0763$ \\
\hline & {$[0,0000]$} & {$[0,0022]$} & {$[0,0530]$} & {$[0,0420]$} & {$[0,0340]$} & {$[0,6207]$} \\
\hline \multirow[t]{2}{*}{$\varphi$} & $-1,3163$ & 0,0001 & $-3,6063$ & 0,0000 & $-7,4719$ & 0,0000 \\
\hline & {$[0,0026]$} & {$[0,2407]$} & {$[0,0625]$} & {$[0,1706]$} & {$[0,0000]$} & {$[0,000]$} \\
\hline \multirow[t]{2}{*}{$\eta$} & $-0,4747$ & $-0,0102$ & 0,4602 & 0,0841 & $-2,2465$ & $-0,0345$ \\
\hline & {$[0,0002]$} & {$[0,9374]$} & {$[0,0203]$} & {$[0,5739]$} & {$[0,0000]$} & {$[0,0000]$} \\
\hline \multirow[t]{2}{*}{$\lambda$} & $-0,5204$ & 0,2913 & $-0,2203$ & 0,4904 & 2,7465 & 0,6629 \\
\hline & {$[0,0000]$} & {$[0,4815]$} & {$[0,1965]$} & {$[0,1953]$} & {$[0,0000]$} & {$[0,0000]$} \\
\hline \multirow[t]{2}{*}{$\theta$} & 0,7918 & 0,6567 & 0,7135 & 0,4731 & 0,1304 & 0,9477 \\
\hline & {$[0,0000]$} & {$[0,0191]$} & {$[0,0000]$} & {$[0,0947]$} & {$[0,4568]$} & {$[0,0000]$} \\
\hline$R^{2}$ & 0,1440 & 0,1564 & 0,0659 & 0,0668 & $-0,1424$ & $-0,0088$ \\
\hline Akaike & $-4,9527$ & $-4,7418$ & $-8,5268$ & $-8,5289$ & $-6,9777$ & $-7,7145$ \\
\hline Schwarz & $-4,7553$ & $-4,5444$ & $-8,3675$ & $-8,3696$ & $-6,7461$ & $-7,4829$ \\
\hline Hannan- & $-4,8746$ & $-4,6637$ & $-8,4624$ & $-8,4645$ & $-6,8898$ & $-7,6266$ \\
\hline
\end{tabular}

Notas. Esta tabla muestra los resultados de regresión que provienen de la estimación de las ecuaciones (9) y (15), siendo el pvalue los valores que están en corchetes. La interpretación del parámetro de asimetría en los modelos EGARCH $(1,1)$ indican que si se satisface que $\lambda<0$ implica que un shock negativo genera mayor volatilidad que un shock positivo. Mientras que la interpretación del parámetro de asimetría en los modelos TGARCH $(1,1)$ indican que si se satisface que $\lambda>0$ implica que para ese activo hay asimetría en las noticias (Shock), es decir, que un shock negativo tiene mayores efectos de volatilidad que un shock positivo. Los datos de las acciones provienen de la bolsa de valores de Ecuador. Elaboración propia.

Para el caso de Ecuador, las estimaciones del parámetro $(\lambda)$ fueron no significativas en los siguientes modelos: i) TGARCH del Banco Guayaquil; ii) EGARCH y TGARCH de Corporación la Favorita iii) EGARCH de Natluk debido a que no se satisface la condición de $\lambda<0$. Por lo tanto, se muestra que la pandemia no afectó a la variabilidad de los precios de estas acciones. La estimación de la varianza condicional en condiciones normales queda construida como:

$$
\begin{gathered}
\widehat{h_{t}} \text { Banco de Guayaquil }=0,0001+0,6567 \widehat{h_{t-1}}+(-0,0102) \widehat{u_{t-1}^{2}} \\
\widehat{h_{t}} \text { Corp. La Favorita }=0,0000+0,4731 \widehat{h_{t-1}}+(0,0841) \widehat{u_{t-1}^{2}} \\
\widehat{h_{t}} \text { Natluk }=0,0000+0,9477 \widehat{h_{t-1}}+(-0,0345) \widehat{u_{t-1}^{2}}
\end{gathered}
$$


Tabla 3

Estimaciones de los parámetros y pruebas de diagnóstico para Perú

\begin{tabular}{|c|c|c|c|c|c|c|}
\hline & $\begin{array}{l}\text { Banco BBVA } \\
\operatorname{EGARCH}(\mathbf{1 , 1})\end{array}$ & $\begin{array}{l}\text { Banco BBVA } \\
\text { TGARCH }(\mathbf{1 , 1})\end{array}$ & $\begin{array}{l}\text { Empresa } \\
\text { Siderúrgica } \\
\text { EGARCH }(\mathbf{1 , 1})\end{array}$ & $\begin{array}{l}\text { Empresa } \\
\text { Siderúrgica } \\
\text { TGARCH }(\mathbf{1 , 1 )}\end{array}$ & $\begin{array}{l}\text { U. Andina } \\
\text { Cementos } \\
\text { EGARCH }(\mathbf{1 , 1})\end{array}$ & $\begin{array}{l}\text { U. Andina } \\
\text { Cementos } \\
\text { TGARCH }(\mathbf{1 , 1})\end{array}$ \\
\hline \multirow[t]{2}{*}{$\mathrm{c}$} & $-0,0039$ & $-0,0021$ & $-0,0017$ & $-0,0024$ & $-0,0037$ & $-0,0016$ \\
\hline & {$[0,0000]$} & {$[0,0814]$} & {$[0,6383]$} & {$[0,0257]$} & {$[0,0000]$} & {$[0,0695]$} \\
\hline \multirow[t]{2}{*}{$\operatorname{Var}(-1)$} & $-0,1414$ & 0,0014 & 0,0788 & 0,0619 & 0,2127 & 0,0295 \\
\hline & {$[0,1489]$} & {$[0,9929]$} & {$[0,4587]$} & {$[0,4735]$} & {$[0,0112]$} & {$[0,8696]$} \\
\hline \multirow[t]{2}{*}{$\varphi$} & $-0,8259$ & 0,0000 & $-4,1861$ & $-0,0000$ & $-0,4794$ & 0,0000 \\
\hline & {$[0,0000]$} & {$[0,0000]$} & {$[0,4586]$} & {$[0,0451]$} & {$[0,0002]$} & {$[0,1336]$} \\
\hline \multirow[t]{2}{*}{$\eta$} & $-0,2240$ & $-0,0786$ & $-0,0862$ & 0,0231 & $-0,3535$ & $-0,0174$ \\
\hline & {$[0,0114]$} & {$[0,0000]$} & {$[0,6576]$} & {$[0,0000]$} & {$[0,0185]$} & {$[0,7296]$} \\
\hline \multirow[t]{2}{*}{$\lambda$} & $-0,5114$ & 0,4958 & $-0,1382$ & $-0,0637$ & $-0,4218$ & 0,8626 \\
\hline & {$[0,0000]$} & {$[0,0000]$} & {$[0,5955]$} & {$[0,0000]$} & {$[0,0004]$} & {$[0,0014]$} \\
\hline \multirow[t]{2}{*}{$\theta$} & 0,8778 & 0,7153 & 0,4186 & 1,0591 & 0,9063 & 0,7520 \\
\hline & {$[0,0000]$} & {$[0,0000]$} & {$[0,5841]$} & {$[0,0000]$} & {$[0,0000]$} & {$[0,0000]$} \\
\hline$R^{2}$ & 0,0198 & $-0,0092$ & 0,0074 & 0,0071 & 0,0200 & $-0,0422$ \\
\hline Akaike & $-5,4392$ & $-5,3338$ & $-4,3227$ & $-4,5147$ & $-5,5054$ & $-5,2234$ \\
\hline Schwarz & $-5,2631$ & $-5,1623$ & $-4,1466$ & $-4,3386$ & $-5,3293$ & $-5,0473$ \\
\hline Hannan- & $-5,3685$ & $-5,2677$ & $-4,2523$ & $-4,4434$ & $-5,4347$ & $-5,1527$ \\
\hline
\end{tabular}

Notas. Esta tabla muestra los resultados de regresión que provienen de la estimación de las ecuaciones (9) y (15), siendo el pvalue los valores que están en corchetes. La interpretación del parámetro de asimetría en los modelos EGARCH $(1,1)$ indican que si se satisface que $\lambda<0$ implica que un shock negativo genera mayor volatilidad que un shock positivo. Mientras que la interpretación del parámetro de asimetría en los modelos TGARCH $(1,1)$ indican que si se satisface que $\lambda>0$ implica que para ese activo hay asimetría en las noticias (Shock), es decir, que un shock negativo tiene mayores efectos de volatilidad que un shock positivo. Los datos de las acciones provienen de la bolsa de valores de Perú. Elaboración propia.

Para el caso del Perú, las estimaciones del parámetro $(\lambda)$ fueron no significativas en el siguiente modelo: i) EGARCH de la empresa Siderúrgica del Perú. Por lo tanto, se muestra que la pandemia afectó a la variabilidad de los precios de las acciones Banco BBVA y Unión Andina de cementos. La estimación de la varianza condicional frente al shock de la pandemia y en condiciones normales queda construida como:

$$
\begin{aligned}
& \widehat{h_{t}} \text { Banco BBVA }_{\text {Covid } 19}=0,000+0,7153 \widehat{h_{t-1}}+(-0,0786+0,4958) \widehat{u_{t-1}^{2}} \\
& \widehat{h_{t}} \text { Banco BBVA }=0,000+0,7153 \widehat{h_{t-1}}+(-0,0786) \widehat{u_{t-1}^{2}} \\
& \widehat{h_{t}} \text { Emp. Siderúrgica }=-0,000+1,0591 \widehat{h_{t-1}}+(0,0231) \widehat{u_{t-1}^{2}} \\
& \widehat{h_{t}} \text { U. Andina de Cementos } \operatorname{Covid}_{19}=0,000+0,7520 \widehat{h_{t-1}}+(-0,0174+0,8626) \widehat{u_{t-1}^{2}} \\
& \widehat{h_{t}} \mathrm{U} . \text { Andina de Cementos }=0,000+0,7520 \widehat{h_{t-1}}+(-0,0174) \widehat{u_{t-1}^{2}}
\end{aligned}
$$


Tabla 4

Estimaciones de los parámetros y pruebas de diagnóstico para Colombia

\begin{tabular}{lcccccc} 
& $\begin{array}{c}\text { Bancolombia } \\
\text { EGARCH (1,1) }\end{array}$ & $\begin{array}{c}\text { Bancolombia } \\
\text { TGARCH (1,1) }\end{array}$ & $\begin{array}{c}\text { Grupo Argos } \\
\text { EGARCH (1,1) }\end{array}$ & $\begin{array}{c}\text { Grupo Argos } \\
\text { TGARCH (1,1) }\end{array}$ & $\begin{array}{c}\text { Grupo Nutresa } \\
\text { EGARCH (1,1) }\end{array}$ & $\begin{array}{c}\text { Grupo Nutresa } \\
\text { TGARCH (1,1) }\end{array}$ \\
\hline $\mathrm{c}$ & $-0,0071$ & $-0,0051$ & $-0,0065$ & $-0,0069$ & $-0,0248$ & $-0,0027$ \\
& {$[0,0013]$} & {$[0,0000]$} & {$[0,0103]$} & {$[0,0365]$} & {$[0,0003]$} & {$[0,0069]$} \\
$\operatorname{Var}(-1)$ & 0,1139 & $-0,0378$ & 0,0935 & 0,2097 & $-0,2301$ & $-0,1101$ \\
& {$[0,3491]$} & {$[0,7811]$} & {$[0,4305]$} & {$[0,1065]$} & {$[0,0043]$} & {$[0,2086]$} \\
$\varphi$ & $-0,3524$ & 0,0000 & $-0,6830$ & 0,0000 & $-0,1179$ & 0,0000 \\
& {$[0,0987]$} & {$[0,0000]$} & {$[0,0012]$} & {$[0,1267]$} & {$[0,3731]$} & {$[0,0000]$} \\
$\eta$ & $-0,2864$ & $-0,2448$ & 0,4728 & 0,2015 & $-0,1916$ & $-0,1538$ \\
& {$[0,0082]$} & {$[0,0000]$} & {$[0,0005]$} & {$[0,1683]$} & {$[0,1239]$} & {$[0,0031]$} \\
$\lambda$ & $-0,4404$ & 0,3966 & $-0,1429$ & 0,2770 & $-0,2565$ & 0,2818 \\
& {$[0,0001]$} & {$[0,0000]$} & {$[0,1946]$} & {$[0,3261]$} & {$[0,0001]$} & {$[0,0000]$} \\
$\theta$ & 0,9118 & 1,0326 & 0,9472 & 0,6823 & 0,9616 & 1,0043 \\
& {$[0,0000]$} & {$[0,0000]$} & {$[0,0000]$} & {$[0,0000]$} & {$[0,0000]$} & {$[0,0000]$} \\
$R^{2}$ & 0,0161 & $-0,00142$ & 0,0404 & 0,0673 & $-0,0971$ & $-0,0349$ \\
Akaike & $-4,1684$ & $-4,1300$ & $-3,5924$ & $-3,5443$ & $-5,0996$ & $-5,0012$ \\
Schwarz & $-4,0061$ & $-3,9677$ & $-3,4301$ & $-3,3820$ & $-4,9373$ & $-4,8389$ \\
Hannan- & $-4,1028$ & $-4,0644$ & $-3,5368$ & $-3,4788$ & $-5,0340$ & $-4,0367$ \\
Quinn & & & & & &
\end{tabular}

Notas. Esta tabla muestra los resultados de regresión que provienen de la estimación de las ecuaciones (9) y (15), siendo el pvalue los valores que están en corchetes. La interpretación del parámetro de asimetría en los modelos EGARCH $(1,1)$ indican que si se satisface que $\lambda<0$ implica que un shock negativo genera mayor volatilidad que un shock positivo. Mientras que la interpretación del parámetro de asimetría en los modelos TGARCH $(1,1)$ indican que si se satisface que $\lambda>0$ implica que para ese activo hay asimetría en las noticias (Shock), es decir, que un shock negativo tiene mayores efectos de volatilidad que un shock positivo. Los datos de las acciones provienen de la bolsa de valores de Colombia. Elaboración propia.

Para el caso de Colombia, las estimaciones del parámetro $(\lambda)$ fueron no significativas en los siguientes modelos: i) EGARCH y TGACRH del Grupos Argos. Por lo tanto, se muestra que la pandemia afectó a la variabilidad de los precios de las acciones Bancolombia y Grupo Nutresa. La estimación de la varianza condicional frente al shock de la pandemia y en condiciones normales queda construida como:

$$
\begin{aligned}
& \widehat{h_{t}} \text { Bancolombia }_{\text {Covid } 19}=0,0000+1,0326 \widehat{h_{t-1}}+(-0,2448+0,3966) \widehat{u_{t-1}^{2}} \\
& \widehat{h_{t}} \text { Bancolombia }=0,000+1,0326 \widehat{h_{t-1}}+(-0,2448) \widehat{u_{t-1}^{2}} \\
& \widehat{h_{t}} \text { Grupo Argos }=0,0000+0,68231 \widehat{h_{t-1}}+(0,2015) \widehat{u_{t-1}^{2}} \\
& \widehat{h_{t}} \text { Grupo Nutresa }{ }_{\text {Covid } 19}=0,0000+1,0043 \widehat{h_{t-1}}+(-0,1538+0,2818) \overline{u_{t-1}^{2}} \\
& \widehat{h_{t}} \text { Grupo Nutresa }=0,0000+1,0043 \widehat{h_{t-1}}+(-0,1538) \widehat{u_{t-1}^{2}}
\end{aligned}
$$


Tabla 5

Estimaciones de los parámetros y pruebas de diagnóstico para Argentina

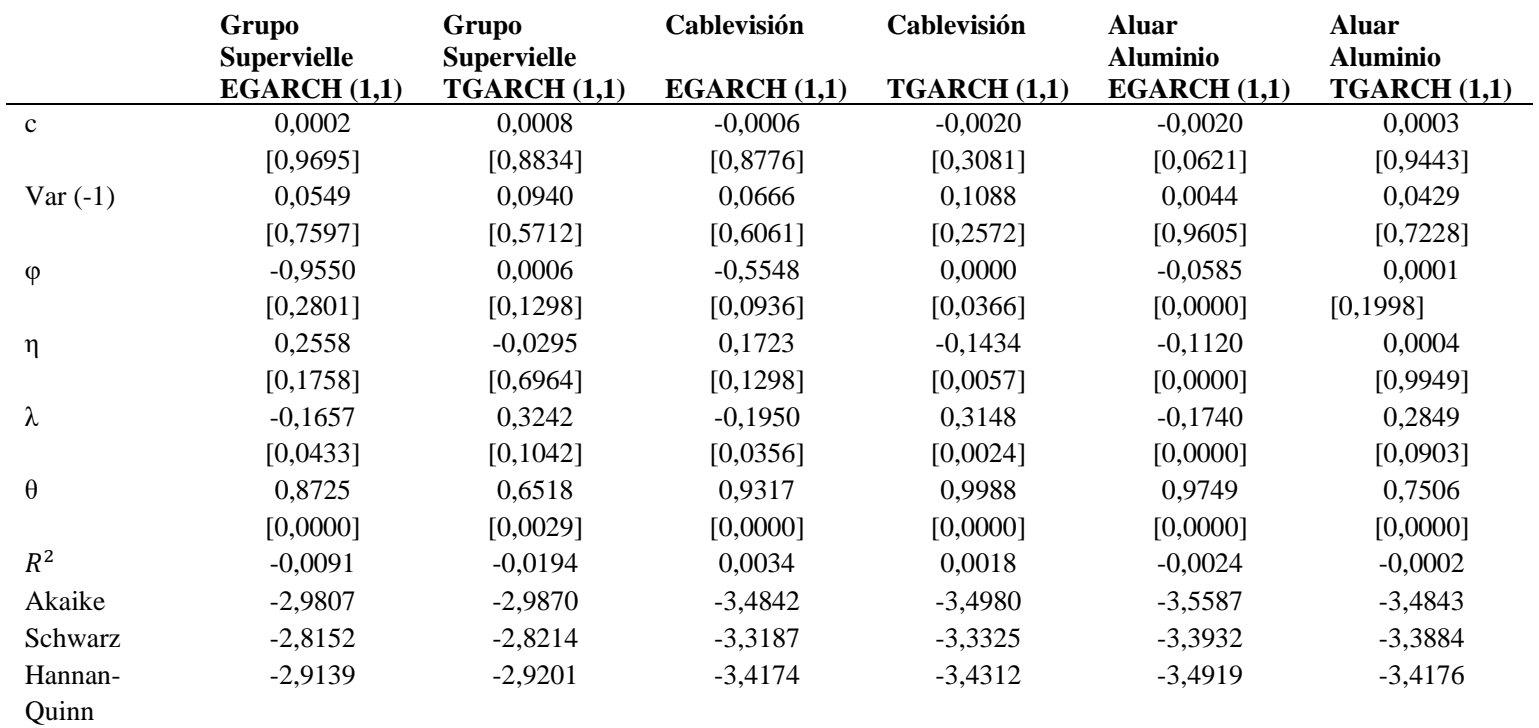

Notas. Esta tabla muestra los resultados de regresión que provienen de la estimación de las ecuaciones (9) y (15), siendo el pvalue los valores que están en corchetes. La interpretación del parámetro de asimetría en los modelos EGARCH $(1,1)$ indican que si se satisface que $\lambda<0$ implica que un shock negativo genera mayor volatilidad que un shock positivo. Mientras que la interpretación del parámetro de asimetría en los modelos TGARCH $(1,1)$ indican que si se satisface que $\lambda>0$ implica que para ese activo hay asimetría en las noticias (Shock), es decir, que un shock negativo tiene mayores efectos de volatilidad que un shock positivo. Los datos de las acciones provienen de la bolsa de valores Argentina. Elaboración propia.

Para el caso de Argentina, las estimaciones del parámetro ( $\lambda$ ) fueron no significativas en los siguientes modelos: i) TGARCH del Grupo Superville; ii) TGARCH de Aluar Aluminio. Por lo tanto, se muestra que la pandemia afectó a la variabilidad del precio de la acción de Cablevisión. La estimación de la varianza condicional frente al shock de la pandemia y en condiciones normales queda construida como:

$$
\begin{gathered}
\widehat{h_{t}} \text { Grupo Supervielle }=0,0006+0,6518 \widehat{h_{t-1}}+(-0,0295) \widehat{u_{t-1}^{2}} \\
\widehat{h_{t}} \text { Cablevisión }_{\text {Covid } 19}=0,0000+0,9988 \widehat{h_{t-1}}+(-0,1434+0,3148) \widehat{u_{t-1}^{2}} \\
\widehat{h_{t}} \text { Cablevisión }=0,0000+0,99881 \widehat{h_{t-1}}+(-0,1434) \widehat{u_{t-1}^{2}} \\
\widehat{h_{t}} \text { Aluar Aluminio }=0,0001+0,7506 \widehat{h_{t-1}}+(0,0004) \widehat{u_{t-1}^{2}}
\end{gathered}
$$


Tabla 6

Estimaciones de los parámetros y pruebas de diagnóstico para Chile

\begin{tabular}{|c|c|c|c|c|c|c|}
\hline & $\begin{array}{l}\text { Itau Corp } \\
\text { Bank } \\
\text { EGARCH }(1,1)\end{array}$ & $\begin{array}{l}\text { Itau Corp } \\
\text { Bank } \\
\text { TGARCH }(1,1)\end{array}$ & $\begin{array}{l}\text { Falabella } \\
\text { EGARCH }(1,1)\end{array}$ & $\begin{array}{l}\text { Falabella } \\
\operatorname{TGARCH}(1,1)\end{array}$ & $\begin{array}{l}\text { Embotelladora } \\
\text { Andina } \\
\text { EGARCH }(1,1)\end{array}$ & $\begin{array}{l}\text { Embotelladora } \\
\text { Andina } \\
\text { TGARCH }(1,1)\end{array}$ \\
\hline \multirow[t]{2}{*}{$\mathrm{c}$} & $-0,0125$ & $-0,0107$ & $-0,0052$ & $-0,0027$ & $-0,0018$ & $-0,0030$ \\
\hline & {$[0,0000]$} & {$[0,0002]$} & {$[0,2373]$} & {$[0,1211]$} & {$[0,6096]$} & 0,1502 \\
\hline \multirow[t]{2}{*}{$\operatorname{Var}(-1)$} & $-0,1370$ & $-0,2509$ & 0,0717 & $-0,1029$ & $-0,1947$ & $-0,2182$ \\
\hline & {$[0,2303]$} & {$[0,0553]$} & {$[0,1121]$} & {$[0,4393]$} & {$[0,1704]$} & {$[0,0824]$} \\
\hline \multirow[t]{2}{*}{$\varphi$} & $-0,3054$ & 0,0000 & $-8,2012$ & 0,0000 & $-8,0725$ & 0,0000 \\
\hline & {$[0,1051]$} & {$[0,0474]$} & {$[0,0000]$} & {$[0,7212]$} & {$[0,0011]$} & {$[0,4123]$} \\
\hline \multirow[t]{2}{*}{$\eta$} & $-0,0943$ & $-0,1231$ & $-0,5035$ & 0,1761 & 0,5578 & 0,1365 \\
\hline & {$[0,4619]$} & {$[0,1933]$} & {$[0,0584]$} & {$[0,1555]$} & {$[0,0326]$} & {$[0,3198]$} \\
\hline \multirow[t]{2}{*}{$\lambda$} & $-0,4336$ & 0,5452 & 0,5327 & 0,6497 & 0,4242 & 0,4622 \\
\hline & {$[0,0000]$} & {$[0,0003]$} & {$[0,0044]$} & {$[0,0231]$} & {$[0,0113]$} & {$[0,0211]$} \\
\hline \multirow[t]{2}{*}{$\theta$} & 0,9394 & 0,83331 & $-0,2868$ & 0,7151 & $-0,1110$ & 0,7377 \\
\hline & {$[0,0000]$} & {$[0,0000]$} & {$[0,0549]$} & {$[0,0000]$} & {$[0,7555]$} & {$[0,0000]$} \\
\hline$R^{2}$ & 0,0716 & 0,1079 & 0,0001 & $-0,0220$ & 0,1164 & 0,1235 \\
\hline Akaike & $-4,1417$ & $-4,0447$ & $-3,6334$ & $-3,9527$ & $-3,9471$ & $-4,2944$ \\
\hline Schwarz & $-3,9814$ & $-3,8844$ & $-3,4731$ & $-3,7924$ & $-3,7866$ & $-4,1341$ \\
\hline Hannan- & $-4,0769$ & $-3,9799$ & $-3,5686$ & $-3,8879$ & $-3,8823$ & $-4,2296$ \\
\hline
\end{tabular}

Notas. Esta tabla muestra los resultados de regresión que provienen de la estimación de las ecuaciones (9) y (15), siendo el pvalue los valores que están en corchetes. La interpretación del parámetro de asimetría en los modelos EGARCH $(1,1)$ indican que si se satisface que $\lambda<0$ implica que un shock negativo genera mayor volatilidad que un shock positivo. Mientras que la interpretación del parámetro de asimetría en los modelos TGARCH $(1,1)$ indican que si se satisface que $\lambda>0$ implica que para ese activo hay asimetría en las noticias (Shock), es decir, que un shock negativo tiene mayores efectos de volatilidad que un shock positivo. Los datos de las acciones provienen de la bolsa de valores de Chile. Elaboración propia.

Para el caso de Chile, las estimaciones del parámetro $(\lambda)$ fueron no significativas en los siguientes modelos: i) EGARCH de Falabella debido a que no se satisface la condición de $\lambda<0$; ii) EGARCH de Embotelladora Andina debido a que tampoco satisface la condición de $\lambda<0$. Por lo tanto, se muestra que la pandemia afectó solo a la variabilidad del precio de la acción de Itaú Corp Bank. La estimación de la varianza condicional frente al shock de la pandemia y en condiciones normales queda construida como:

$$
\begin{aligned}
& \widehat{h_{t}} \text { Itaú Corp Bank } \mathrm{Covid}_{19}=0,0000+0,8331 \widehat{h_{t-1}}+(-0,1231+0,5452) \widehat{u_{t-1}^{2}} \\
& \widehat{h_{t}} \text { Itaú Corp Bank }=0,0000+0,8331 \widehat{h_{t-1}}+(-0,1231) \widehat{u_{t-1}^{2}} \\
& \widehat{h_{t}} \text { Falabella }=0,0000+0,7151 \widehat{h_{t-1}}+(0,1761) \widehat{u_{t-1}^{2}} \\
& \widehat{h_{t}} \text { Embotelladora Andina }=0,0000+0,7377 \widehat{h_{t-1}}+(0,1365) \widehat{u_{t-1}^{2}}
\end{aligned}
$$


Tabla 7

Estimaciones de los parámetros y pruebas de diagnóstico para México

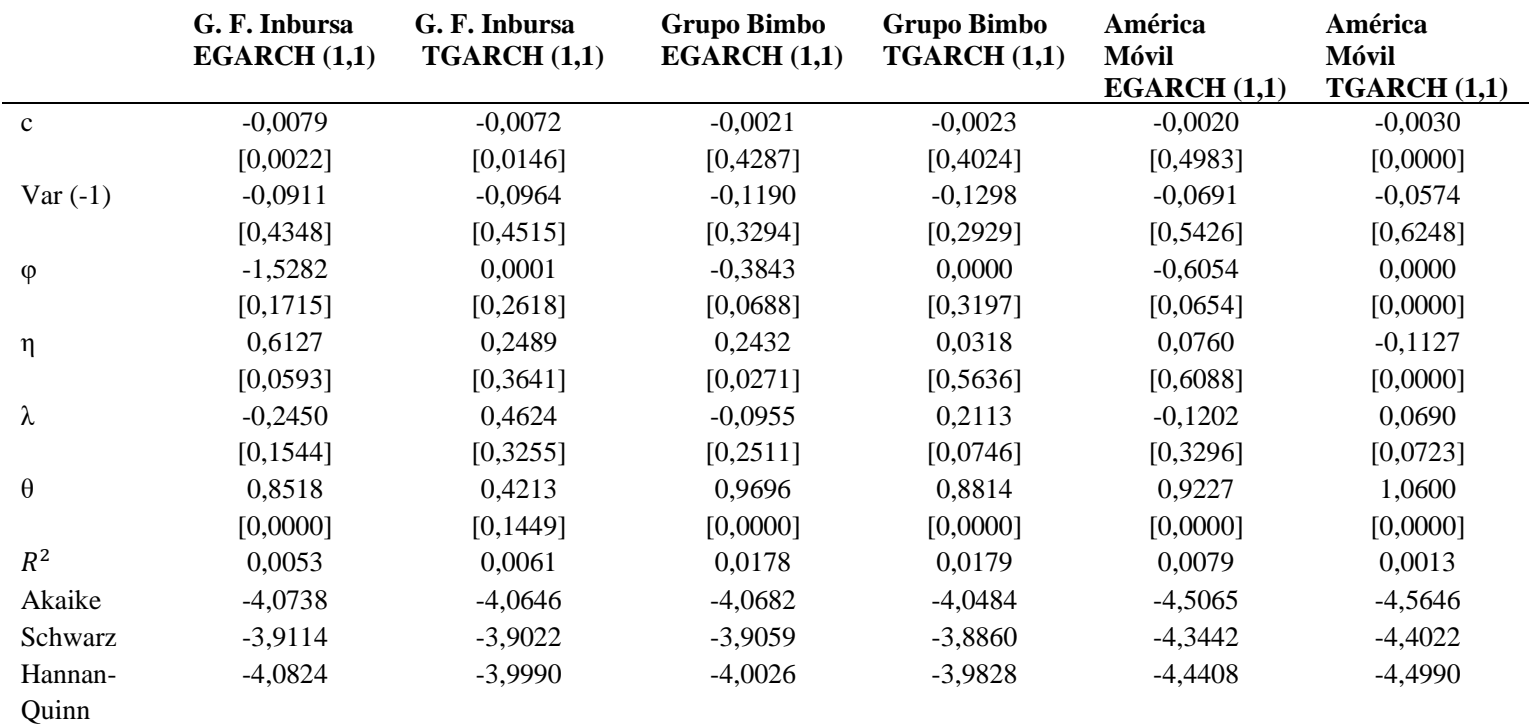

Notas. Esta tabla muestra los resultados de regresión que provienen de la estimación de las ecuaciones (9) y (15), siendo el pvalue los valores que están en corchetes. La interpretación del parámetro de asimetría en los modelos EGARCH $(1,1)$ indican que si se satisface que $\lambda<0$ implica que un shock negativo genera mayor volatilidad que un shock positivo. Mientras que la interpretación del parámetro de asimetría en los modelos TGARCH $(1,1)$ indican que si se satisface que $\lambda>0$ implica que para ese activo hay asimetría en las noticias (Shock), es decir, que un shock negativo tiene mayores efectos de volatilidad que un shock positivo. Los datos de las acciones provienen de la bolsa de valores de México. Elaboración propia.

Para el caso de México, las estimaciones del parámetro $(\lambda)$ fueron no significativas en todos los modelos. Por lo tanto, se muestra que la pandemia no afectó a la variabilidad de los precios de las acciones de: Grupo Financiero Inbursa, Grupo Bimbo y América móvil. La estimación de la varianza condicional en condiciones normales queda construida como:

$$
\begin{aligned}
\widehat{h_{t}} \text { Grupo F. Inbursa } & =0,0001+0,4213 \widehat{h_{t-1}}+(0,2489) \widehat{u_{t-1}^{2}} \\
\widehat{h_{t}} \text { Grupo Bimbo } & =0,0000+0,8814 \widehat{h_{t-1}}+(0,0318) \widehat{u_{t-1}^{2}} \\
\widehat{h_{t}} \text { América Móvil } & =0,0000+1,0600 \widehat{h_{t-1}}+(-0,1127) \widehat{u_{t-1}^{2}}
\end{aligned}
$$


Tabla 8

Estimaciones de los parámetros y pruebas de diagnóstico para Brasil

\begin{tabular}{|c|c|c|c|c|c|c|}
\hline & $\begin{array}{l}\text { Banco Bradesco } \\
\text { EGARCH }(\mathbf{1 , 1})\end{array}$ & $\begin{array}{r}\text { Banco Bradesco } \\
\text { TGARCH }(1,1)\end{array}$ & $\begin{array}{l}\text { Metalúrgica } \\
\text { Gerdau } \\
\text { EGARCH }(1,1)\end{array}$ & $\begin{array}{l}\text { Metalúrgica } \\
\text { Gerdau } \\
\text { TGARCH }(\mathbf{1 , 1})\end{array}$ & $\begin{array}{l}\text { Atacadao } \\
\text { EGARCH }(1,1)\end{array}$ & $\begin{array}{l}\text { Atacadao } \\
\text { TGARCH }(\mathbf{1 , 1})\end{array}$ \\
\hline $\mathrm{c}$ & $\begin{array}{c}-0,0099 \\
{[0,0001]}\end{array}$ & $\begin{array}{l}-0,0080 \\
{[0,0019]}\end{array}$ & $\begin{array}{l}-0,0016 \\
{[0,7083]}\end{array}$ & $\begin{array}{l}-0,0020 \\
{[0,6887]}\end{array}$ & $\begin{array}{l}-0,0084 \\
{[0,0042]}\end{array}$ & $\begin{array}{c}-0,0091 \\
{[0,0000]}\end{array}$ \\
\hline $\operatorname{Var}(-1)$ & $\begin{array}{l}-0,0101 \\
{[0,9269]}\end{array}$ & $\begin{array}{c}0,0047 \\
{[0,9646]}\end{array}$ & $\begin{array}{c}-0,1534 \\
{[0,1571]}\end{array}$ & $\begin{array}{l}-0,0079 \\
{[0,9267]}\end{array}$ & $\begin{array}{c}-0,0601 \\
{[0,5624]}\end{array}$ & $\begin{array}{c}-0,0616 \\
{[0,4259]}\end{array}$ \\
\hline$\varphi$ & $\begin{array}{c}-0,6369 \\
{[0,0429]}\end{array}$ & $\begin{array}{c}0,0000 \\
{[0,0513]}\end{array}$ & $\begin{array}{c}-0,3701 \\
{[0,0000]}\end{array}$ & $\begin{array}{c}0,0002 \\
{[0,0008]}\end{array}$ & $\begin{array}{l}-1,2289 \\
{[0,1379]}\end{array}$ & $\begin{array}{c}0,0001 \\
{[0,016]}\end{array}$ \\
\hline$\eta$ & $\begin{array}{c}0,1737 \\
{[0,2383]}\end{array}$ & $\begin{array}{c}-0,0789 \\
{[0,4709]}\end{array}$ & $\begin{array}{c}-0,2211 \\
{[-0,0006]}\end{array}$ & $\begin{array}{c}-0,2946 \\
0,0003\end{array}$ & $\begin{array}{c}0,3685 \\
{[0,1876]}\end{array}$ & $\begin{array}{r}-0,1443 \\
{[0,038]}\end{array}$ \\
\hline$\lambda$ & $\begin{array}{c}-0,3936 \\
{[0,0025]}\end{array}$ & $\begin{array}{c}0,6422 \\
{[0,0483]}\end{array}$ & $\begin{array}{c}-0,3152 \\
{[0,0000]}\end{array}$ & $\begin{array}{c}0,4302 \\
{[0,0001]}\end{array}$ & $\begin{array}{c}-0,4391 \\
{[0,0046]}\end{array}$ & $\begin{array}{c}1,1299 \\
{[0,0166]}\end{array}$ \\
\hline$\theta$ & $\begin{array}{c}0,9202 \\
{[0,0000]}\end{array}$ & $\begin{array}{c}0,7856 \\
{[0,0000]}\end{array}$ & $\begin{array}{c}0,9101 \\
{[0,0000]}\end{array}$ & $\begin{array}{c}0,9587 \\
{[0,0000]}\end{array}$ & $\begin{array}{c}0,8662 \\
{[0,0000]}\end{array}$ & $\begin{array}{c}0,6570 \\
{[0,0000]}\end{array}$ \\
\hline$R^{2}$ & 0,0001 & $-0,0038$ & 0,0163 & $-0,0020$ & 0,0063 & 0,0009 \\
\hline Akaike & $-3,8646$ & $-3,8333$ & $-3,0912$ & $-3,1226$ & $-4,2569$ & $-4,2873$ \\
\hline Schwarz & $-3,7023$ & $-3,6710$ & $-2,9289$ & $-2,9602$ & $-4,0946$ & $-4,1250$ \\
\hline $\begin{array}{l}\text { Hannan- } \\
\text { Quinn }\end{array}$ & $-3,7991$ & $-3,7678$ & $-3,0257$ & $-3,0570$ & $-4,1913$ & $-4,2217$ \\
\hline
\end{tabular}

Notas. Esta tabla muestra los resultados de regresión que provienen de la estimación de las ecuaciones (9) y (15), siendo el pvalue los valores que están en corchetes. La interpretación del parámetro de asimetría en los modelos EGARCH $(1,1)$ indican que si se satisface que $\lambda<0$ implica que un shock negativo genera mayor volatilidad que un shock positivo. Mientras que la interpretación del parámetro de asimetría en los modelos TGARCH $(1,1)$ indican que si se satisface que $\lambda>0$ implica que para ese activo hay asimetría en las noticias (Shock), es decir, que un shock negativo tiene mayores efectos de volatilidad que un shock positivo. Los datos de las acciones provienen de la bolsa de valores de Brasil. Elaboración propia.

Para el caso de Brasil, las estimaciones del parámetro $(\lambda)$ fueron significativas e indican que la pandemia afectó a la variabilidad de los precios de las acciones Banco Bradesco, Metalúrgica Gerdau y Atacadao. La estimación de la varianza condicional frente al shock de la pandemia y en condiciones normales queda construida como:

$$
\begin{gathered}
\widehat{h_{t}} \text { Banco Bradesco }{ }_{\text {Covid } 19}=0,0000+0,7856 \widehat{h_{t-1}}+(-0,0789+0,6422) \widehat{u_{t-1}^{2}} \\
\widehat{h_{t}} \text { Banco Bradesco }=0,0000+0,7856 \widehat{h_{t-1}}+(-0,0789) \widehat{u_{t-1}^{2}} \\
\widehat{h_{t}} \text { Metalúrgica Gerdau }{ }_{\text {Covid } 19}=0,0002+0,9587 \widehat{h_{t-1}}+(-0,2946+0,4302) \widehat{u_{t-1}^{2}} \\
\widehat{h_{t}} \text { Metalúrgica Gerdau }=0,0001+0,9587 \widehat{h_{t-1}}+(-0,2946) \widehat{u_{t-1}^{2}} \\
\widehat{h_{t}} \text { Atacadao }_{\text {Covid } 19}=0,0001+0,6570 \widehat{h_{t-1}}+(-0,1443+1,1299) \widehat{u_{t-1}^{2}} \\
\widehat{h_{t}} \text { Atacadao }=0,0000+0,6570 \widehat{h_{t-1}}+(-0,1443) \widehat{u_{t-1}^{2}}
\end{gathered}
$$

Los resultados presentados cuantifican la transmisión de volatilidad de la pandemia COVID19 a las acciones de empresas de Sudamérica, México y Estados Unidos. 
Figura 2

Evolución de precios de las acciones de Sudamérica, México y Estados Unidos

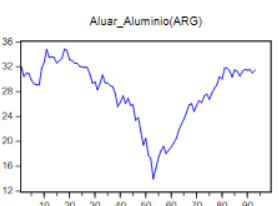

anco_de_Guayaquil|EOU

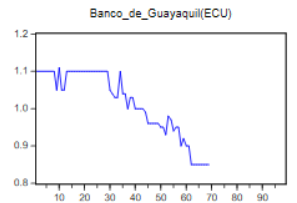

Empres___siderurgica_del_Peru(PERU)

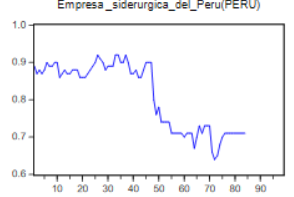

Grupo_Financiero_Inbursa(Mer)

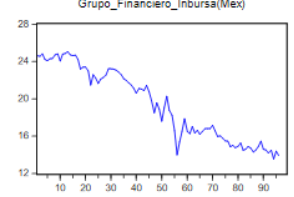

Natukeche

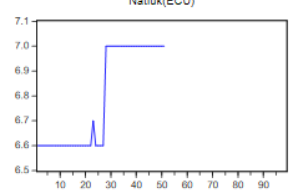

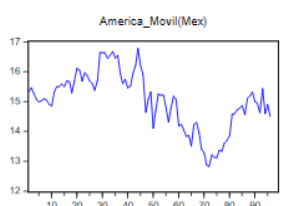

Bancolombia/COL)

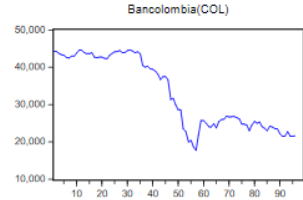

Falabella(Chile)

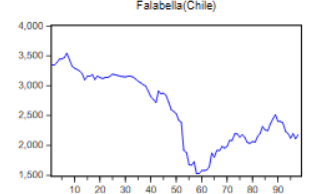

Grupo_Nutress_(COL)

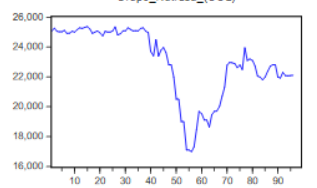

Metalurgica_Gerduu(Bra)

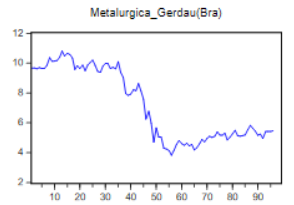

Atacadoo(Bra)

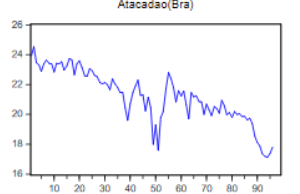

Cablevision(ARG)

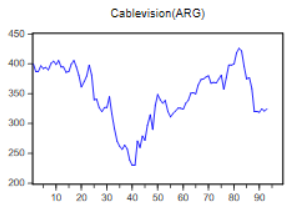

Genera!_Motors(EEUU)

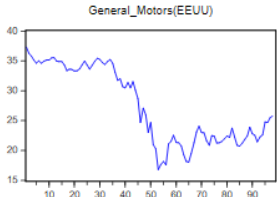

Grupo_Supervielle_(ARG)

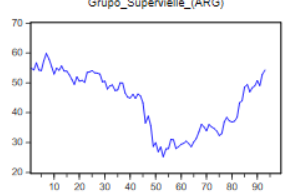

Union_Andina_de_Cementos_UNACEM(PERU)

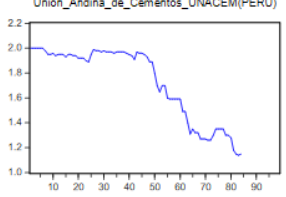

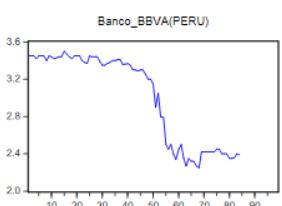
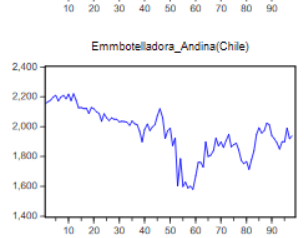

Grupo_Argos(COL)

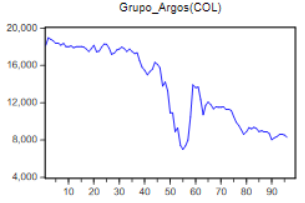

trau_Corp_Bank|Chile

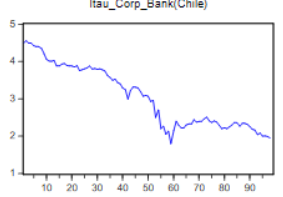

Waimart(EEUU)

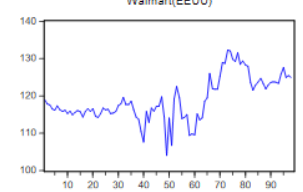

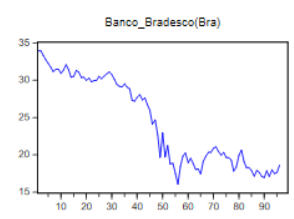

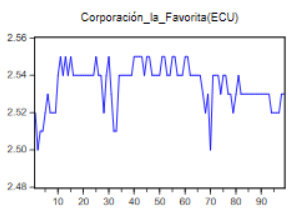

Grupo_Bimbo(Mex)

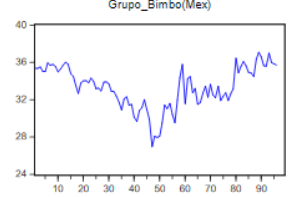

JPMorgan_Chase(EEUU)

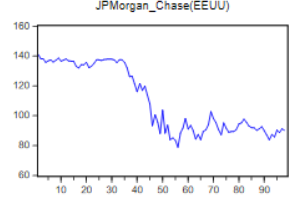

${ }_{10}^{1} \frac{1}{20}+\frac{1}{30}+\frac{1}{40}+\frac{1}{1}+\frac{1}{1}+\frac{1}{1}+\frac{1}{1}+\frac{1}{10}$

Notas. La figura muestra la evolución de precios entre el 5 de enero hasta el 20 de mayo de 2020. El estudio considera el análisis de las acciones con mayor presencia bursátil de cada sector de los países que se mencionan a continuación. Estados Unidos: JPMorgan Chase, Walmart, General Motors; Ecuador: Banco de Guayaquil, Corporación la Favorita, Natluk; Perú: Banco BBVA, Empresa Siderúrgica del Perú, Unión Andina de Cementos; Colombia: Bancolombia, Grupo Argos, Grupo Nutresa; Argentina: Grupo Supervielle, Cablevisión, Aluar Aluminio; Chile: Banco Itaú, Falabella, Embotelladora Andina; México: Grupo Financiero Inbursa, Grupo Bimbo, América móvil; Brasil: Banco Bradesco, Metalúrgica Gerdau, Atacadao. Fuente: obtenidas de las bolsas de valores de cada país. Elaboración propia. 
Figura 3

Rendimiento diario de las acciones de Sudamérica, México y Estados Unidos
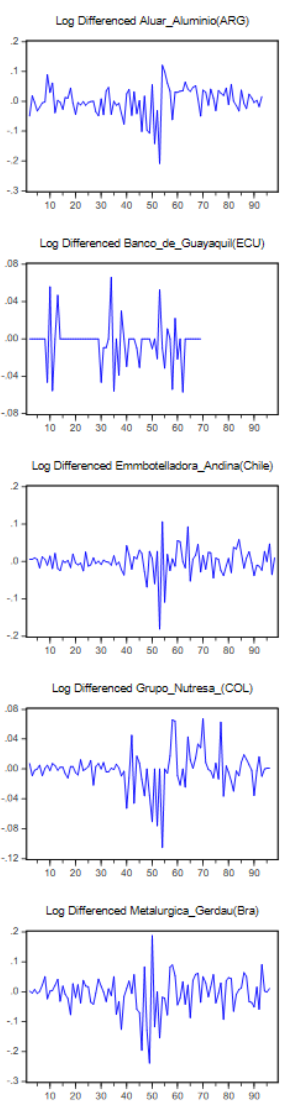

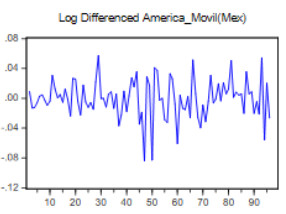

Log Differencesed Bancolombial (COL)

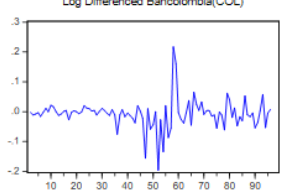

Log Differenced Falabelal(Chile)
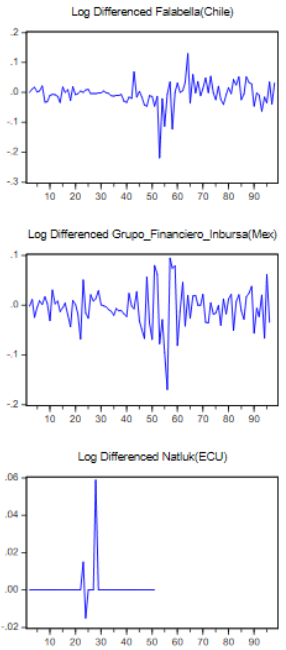

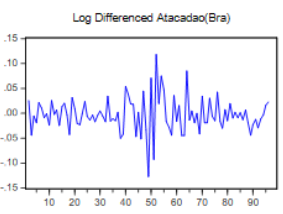

Log Difiererenced Cabolevision(ARG)

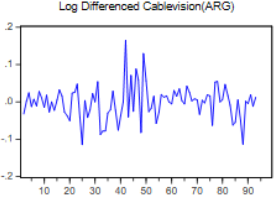

Log Differenosed Generai Motors (EEUU)
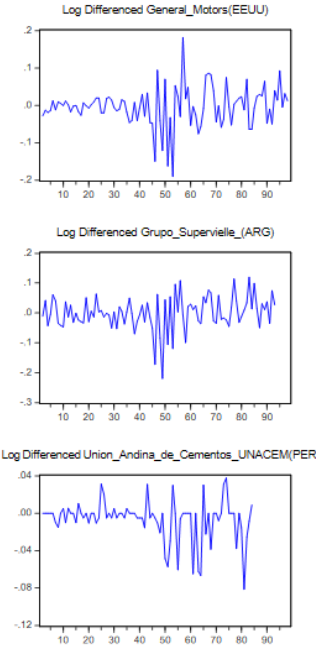
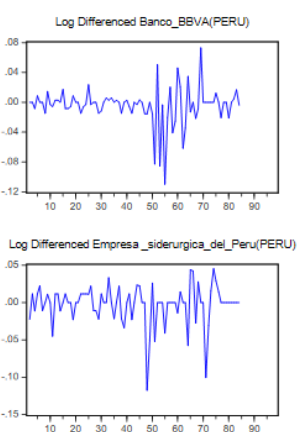

Log Differenced Grupo-Across $(\mathrm{CO})$

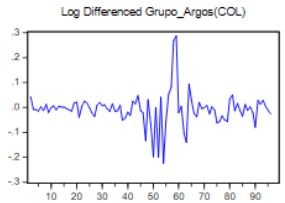

Log Differenced hau_Corp__Bank(Chile)

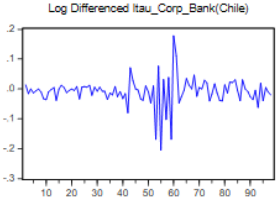

Log Differencoed Walmatt(EEUU

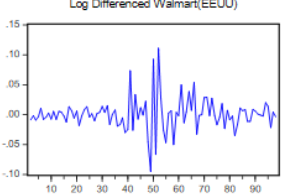

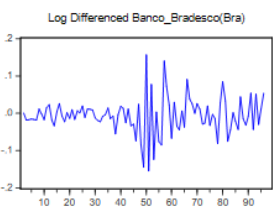
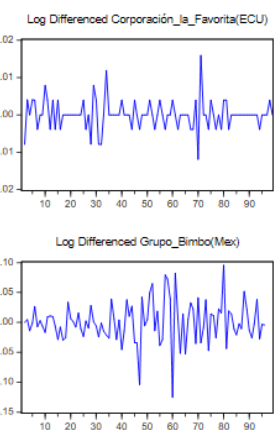

Log Differenced JPMorgan_Chasee EEUU

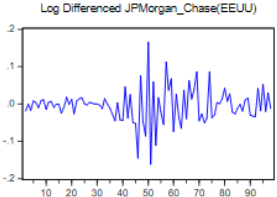

Notas. La figura muestra el rendimiento diario de las acciones entre el 5 de enero hasta el 20 de mayo de 2020. El estudio considera el análisis de las acciones con mayor presencia bursátil de cada sector de los países que se mencionan a continuación. Estados Unidos: JPMorgan Chase, Walmart, General Motors; Ecuador: Banco de Guayaquil, Corporación la Favorita, Natluk; Perú: Banco BBVA, Empresa Siderúrgica del Perú, Unión Andina de Cementos; Colombia: Bancolombia, Grupo Argos, Grupo Nutresa; Argentina: Grupo Supervielle, Cablevisión, Aluar Aluminio; Chile: Banco Itaú, Falabella, Embotelladora Andina; México: Grupo Financiero Inbursa, Grupo Bimbo, América móvil; Brasil: Banco Bradesco, Metalúrgica Gerdau, Atacadao. Fuente: obtenidas de las bolsas de valores de cada país. Elaboración propia. 
Figura 4

Distribución de frecuencia de las acciones de Sudamérica, México y Estados Unidos
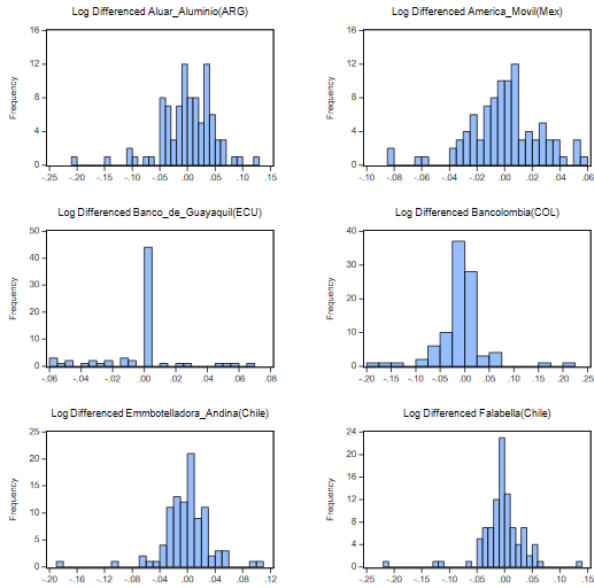

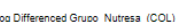
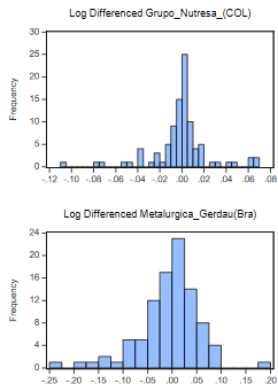

Log Differenoed Bancolombia(COOS)

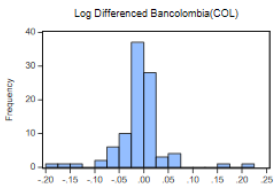

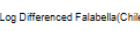
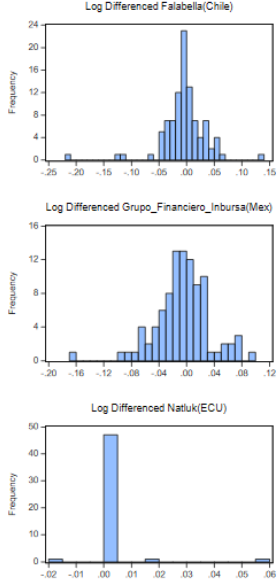

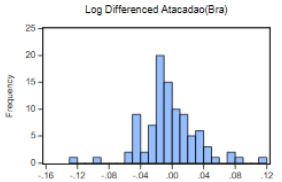

Log Differencesed Cablewision(AR

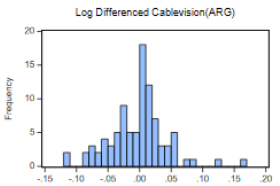

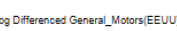

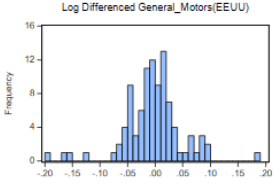

Log Diterencest Gon
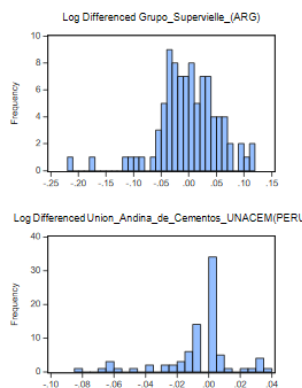
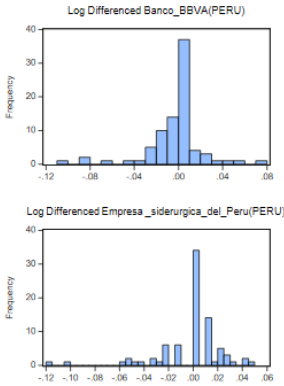

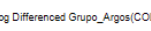

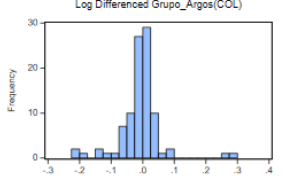

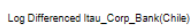
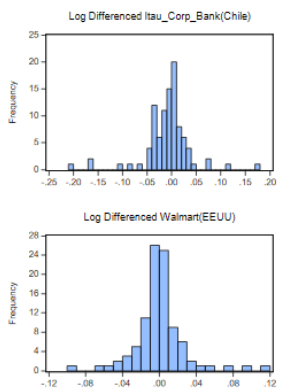
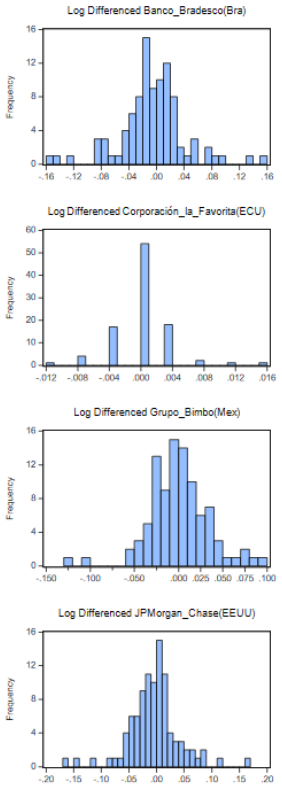

\section{(1)}

Notas. Representación gráfica de los intervalos en el eje X y las frecuencias relativas en el eje Y. La figura muestra la distribución de frecuencia de las acciones en el periodo comprendido entre el 5 de enero hasta el 20 de mayo de 2020 . El estudio considera el análisis de $\mathrm{n}$ acciones con mayor presencia bursátil de cada sector de los países que se mencionan a continuación. Estados Unidos: JPMorgan Chase, Walmart, General Motors; Ecuador: Banco de Guayaquil, Corporación la Favorita, Natluk; Perú: Banco BBVA, Empresa Siderúrgica del Perú, Unión Andina de Cementos; Colombia: Bancolombia, Grupo Argos, Grupo Nutresa; Argentina: Grupo Supervielle, Cablevisión, Aluar Aluminio; Chile: Banco Itaú, Falabella, Embotelladora Andina; México: Grupo Financiero Inbursa, Grupo Bimbo, América móvil; Brasil: Banco Bradesco, Metalúrgica Gerdau, Atacadao. Fuente: obtenidas de las bolsas de valores de cada país. Elaboración propia.

\section{Tabla 9}

Pruebas de raíz unitaria

\begin{tabular}{lcccc}
\multicolumn{1}{c}{ Método } & Estadístico & Prob** & $\begin{array}{c}\text { Secciones } \\
\text { cruzadas }\end{array}$ & Obs \\
\hline $\begin{array}{l}\text { Ho: proceso raíz unitario conjunto. } \\
\text { Levin, Lin \& Chu t* }\end{array}$ & -52.4277 & 0,0000 & 24 & 2149 \\
Ho: proceso raíz unitario individual & & & & \\
Im, Pesaran y Shin W-stat & -48.3182 & 0,0000 & 24 & 2149 \\
ADF - Fisher Chi cuadrado & 677.184 & 0,0000 & 24 & 2149 \\
PP - Fisher Chi cuadrado & 671.660 & 0,0000 & 24 & 2154 \\
\hline
\end{tabular}

Notas. **Las probabilidades para las pruebas de Fisher se calculan utilizando una distribución asintótica de chi cuadrado. Todas las demás pruebas asumen normalidad asintótica. Se observa que el estadístico de prueba de Dickey-Fuller Aumentado en valor absoluto es 677.184, el estadístico PP con valor absoluto es 671.660 y el estadístico Pesaran y Shin con valor absoluto es de 48,3182, el p-value es menor al 5\% de nivel de significancia lo cual conduce a rechazar la hipótesis nula, esto indica que las series utilizadas son estacionarias. 